\title{
Presynaptic Dystroglycan-Pikachurin Complex Regulates the Proper Synaptic Connection between Retinal Photoreceptor and Bipolar Cells
}

\author{
Yoshihiro Omori, ${ }^{1,2,3 *}$ Fumiyuki Araki, ${ }^{1,2,4 *}$ Taro Chaya, ${ }^{1,2}$ Naoko Kajimura, ${ }^{5}$ Shoichi Irie, ${ }^{1,2}$ Koji Terada, ${ }^{1}$ \\ Yuki Muranishi, ${ }^{1,2}$ Toshinori Tsujii, ${ }^{1,2}$ Shinji Ueno, ${ }^{6}$ Toshiyuki Koyasu, ${ }^{6}$ Yasuhiro Tamaki, ${ }^{4}$ Mineo Kondo, ${ }^{7}$ \\ Shiro Amano, ${ }^{4}$ and Takahisa Furukawa ${ }^{1,2}$ \\ ${ }^{1}$ Department of Developmental Biology, Osaka Bioscience Institute, ${ }^{2}$ Japan Science and Technology Agency, Core Research for Evolutional Science and \\ Technology, and ${ }^{3}$ Precursory Research for Embryonic Science and Technology, Osaka, 565-0874, Japan, ${ }^{4}$ Department of Ophthalmology, University of \\ Tokyo Graduate School of Medicine, Tokyo, 113-8655, Japan, ${ }^{5}$ Research Center for Ultra-High Voltage Electron Microscopy, Osaka University, Osaka, 567- \\ 0047, Japan, ${ }^{6}$ Department of Ophthalmology, Nagoya University Graduate School of Medicine, Nagoya, 466-8550, Japan, and ${ }^{7}$ Department of \\ Ophthalmology, Mie University Graduate School of Medicine, Mie, 514-8507, Japan
}

Dystroglycan (DG) is a key component of the dystrophin-glycoprotein complex (DGC) at the neuromuscular junction postsynapse. In the mouse retina, the DGC is localized at the presynapse of photoreceptor cells, however, the function of presynaptic DGC is poorly understood. Here, we developed and analyzed retinal photoreceptor-specific $D G$ conditional knock-out $(D G C K O)$ mice. We found that the $D G$ CKO retina showed a reduced amplitude and a prolonged implicit time of the ERG b-wave. Electron microscopic analysis revealed that bipolar dendrite invagination into the photoreceptor terminus is perturbed in the $D G$ CKO retina. In the $D G$ CKO retina, pikachurin, a DG ligand in the retina, is markedly decreased at photoreceptor synapses. Interestingly, in the Pikachurin ${ }^{-1-}$ retina, the DG signal at the ribbon synaptic terminus was severely reduced, suggesting that pikachurin is required for the presynaptic accumulation of DG at the photoreceptor synaptic terminus, and conversely DG is required for pikachurin accumulation. Furthermore, we found that overexpression of pikachurin induces formation and clustering of a DG-pikachurin complex on the cell surface. The Laminin G repeats of pikachurin, which are critical for its oligomerization and interaction with DG, were essential for the clustering of the DG-pikachurin complex as well. These results suggest that oligomerization of pikachurin and its interaction with DG causes DG assembly on the synapse surface of the photoreceptor synaptic terminals. Our results reveal that the presynaptic interaction of pikachurin with DG at photoreceptor terminals is essential for both the formation of proper photoreceptor ribbon synaptic structures and normal retinal electrophysiology.

\section{Introduction}

Dystrophin is an actin-binding cytoskeletal protein, and its mutation in humans causes various types of muscular dystrophy (MD) including Duchenne MD (DMD) and Becker MD (Hoffman

Received Jan. 23, 2012; revised March 6, 2012; accepted March 15, 2012.

Author contributions:Y.O., F.A., Y.T., M.K., S.A., and T.F. designed research;Y.O., F.A., T.C., N.K., S.I., K.T., Y.M., T.T., S.U., T.K., and T.F. performed research; Y.O., F.A., T.C., N.K., S.I, and S.U. analyzed data; Y.O., F.A., T.C., N.K., S.U., and T.F. wrote the paper.

This work was supported by CREST and PRESTO from Japan Science and Technology Agency, a grant for Molecular Brain Science, Grants-in-Aid for Scientific Research on Priority Areas, and Grant-in-Aid for Scientific Research (B), Young Scientists (B); Specially Designated Research Promotion and Scientific Research on Innovative Areas "Intracellular Logistics" from the Ministry of Education, Culture, Sports and Technology of Japan; The Takeda Science Foundation; The Uehara Memorial Foundation; Novartis Foundation; Mochida Memorial Foundation for Medical and Pharmaceutical Research and The Naito Foundation; Senri Life Science Foundation; Kato Memorial Bioscience Foundation; Daiichi-Sankyo Foundation of Life Science; Japanese Retinitis Pigmentosa Society Foundation; and Research Foundation for Opto-Science and Technology. We thank Dr. Kevin Campbell for the DG-flox mouse, and M. Kadowaki, A. Tani, A. Ishimaru, Y. Saioka, H. Abe, and S. Kennedy for technical assistance.

*Y.O. and F.A. contributed equally to this work.

The authors declare no competing financial interests.

Correspondence should be addressed to Takahisa Furukawa, Department of Developmental Biology, 0saka Bioscience Institute, 6-2-4 Furuedai, Suita, 0saka, 565-0874, Japan. E-mail: furukawa@obi.or.jp.

DOI:10.1523/JNEUROSCI.0322-12.2012

Copyright $\odot 2012$ the authors $\quad 0270-6474 / 12 / 326126-12 \$ 15.00 / 0$ and Kunkel, 1989). The dystrophin-glycoprotein complex (DGC) connects the extracellular matrix with the actin cytoskeleton in the neuromuscular junction (NMJ) and in non-muscle tissues including the brain and retina (Henry and Campbell, 1996). Dystroglycan (DG), a key component of the DGC, consists of an extracellular $\alpha$-DG subunit and a transmembrane $\beta$-DG subunit. Several extracellular ligands for $\alpha$-DG, including laminin, agrin, perlecan, and Pikachurin, have been reported (Ibraghimov-Beskrovnaya et al., 1992; Ervasti and Campbell, 1993; Gee et al., 1994; Peng et al., 1998; Sugita et al., 2001; Sato et al., 2008). These DG ligands commonly contain Laminin G repeats, and these domains physically interact with DG. For ligand binding of DG, glycosyltransferase-mediated glycosylation of DG is critical. Mutations of putative glycosyltransferase genes, including Large, Fukutin, FKRP, PoMGnT1, and POMT1 and $P O M T 2$, have been identified in patients with congenital MD (Kobayashi et al., 1998; Brockington et al., 2001; Yoshida et al., 2001; Beltrán-Valero de Bernabé et al., 2002; Longman et al., 2003; van Reeuwijk et al., 2005).

Attenuation of ERGs is observed in both MD patients and their corresponding animal models (Haenggi and Fritschy, 

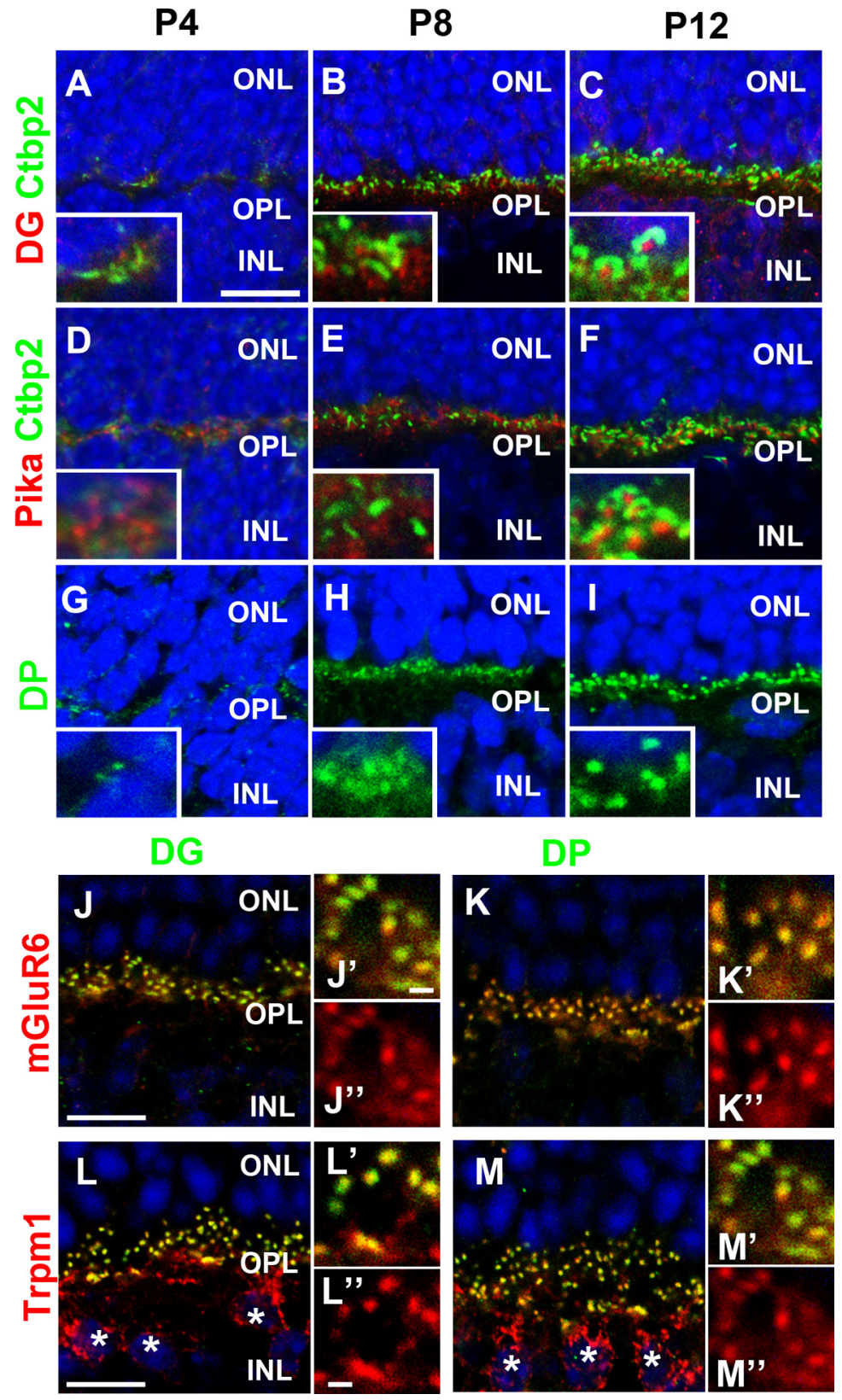

Figure 1. Dystrophin, DG, and Pikachurin are localized at photoreceptor synaptic terminals in the OPL of the developing mouse retina. Retinal sections isolated from $P 4(\boldsymbol{A}, \boldsymbol{D}, \boldsymbol{G}), \mathrm{P} 8(\boldsymbol{B}, \boldsymbol{E}, \boldsymbol{H})$, and $\mathrm{P} 12(\boldsymbol{C}, \boldsymbol{F}, \boldsymbol{I})$ wild-type mice were stained with antibodies against DG (red in $\boldsymbol{A}-\boldsymbol{C}$ ), Pikachurin (red in $\boldsymbol{D}-\boldsymbol{F}$ ), dystrophin (green in $\boldsymbol{G}-\boldsymbol{I}$ ), and (tBP2 (a synaptic ribbon marker, green in $\boldsymbol{A}-\boldsymbol{F}$ ). Higher-magnification views of photoreceptor synapses were shown in the insets. Nuclei were stained with DAPI (blue). DG, Pikachurin, and dystrophin signals were observed in the OPL where photoreceptors form synapses with horizontal and bipolar cells. Retinal sections of adult mice were stained with antibodies against DG (green in $J-J^{\prime \prime}, \boldsymbol{L}-\boldsymbol{L}^{\prime \prime}$ ), dystrophin (green in $\boldsymbol{K}-\boldsymbol{K}^{\prime \prime}, \boldsymbol{M}-\boldsymbol{M}^{\prime \prime}$ ), mGluR6 (red in $J-\boldsymbol{K}^{\prime \prime}$ ), or TRPM1 (red in $\boldsymbol{L}-\boldsymbol{M}^{\prime \prime}$ ). Asterisks indicate TRPM1-positive bipolar cells in the INL $(\boldsymbol{L}, \boldsymbol{M})$. DG and dystrophin signals at photoreceptor synapses were colocalized with mGluR6- and TRPM1-positive bipolar dendritic terminals in the OPL. DG, Pikachurin, and dystrophin signal puncta at photoreceptor ribbon synapses become visible in the OPL after P8. Scale bars: $A, J, L, 10$ $\mu \mathrm{m} ; \boldsymbol{I}^{\prime}, \boldsymbol{L}^{\prime \prime}, 1 \mu \mathrm{m}$.

2006). Reduction of the amplitude of the b-wave response is observed in $80 \%$ of DMD patients (Cibis et al., 1993; Pillers et al., 1993; Fitzgerald et al., 1994; D'Souza et al., 1995). Reduced amplitude and prolonged implicit time of the b-wave, a response of positive polarity originating primarily from the ON-bipolar cells, have been observed in ERGs from various types of MD model mice (Pillers et al., 1995; Lee et al., 2005; Liu et al., 2006). In the retina, DG is found in Müller glial endfeet abutting the inner limiting membrane (ILM) and perivascular glial endfeet as well as photoreceptor synapses in the retinal OPL (Schmitz and Drenckhahn, 1997b; Ueda et al., 2000; Blank et al., 2002). Photoreceptor axonal terminals form ribbon synapses in the OPL, which connect photoreceptor presynapses with the dendritic terminal of both bipolar cells and horizontal cells (tom Dieck and Brandstätter, 2006). We previously reported that an extracellular matrix protein, Pikachurin, is essential for the proper formation of ribbon synaptic structures (Sato et al., 2008). Pikachurin ${ }^{-\prime-}$ mice showed a reduced amplitude and prolonged implicit time of the $b$-wave similar to other mutant mice with perturbed DGC formation. Pikachurin physically interacts with DG, and proper glycosylation of DG is required for its interaction with Pikachurin (Sato et al., 2008; Kanagawa et al., 2010). It was reported that the loss of DG in Müller glial cells causes ERG abnormality (Satz et al., 2009). However, the functional role of presynaptic DG in photoreceptor cells remains unclear.

Here, we report the retinal phenotypes of photoreceptor-specific $D G$ conditional knock-out (DG CKO) mice. Our results show that presynaptic DG-Pikachurin complex is essential for the proper formation of the photoreceptor ribbon synapse and normal retinal electrophysiology.

\section{Materials and Methods}

Generation of the DG CKO mouse. We mated a $D G^{f l o x}$ (Moore et al., 2002) mouse line with a CrxCre transgenic mouse line, which expresses Cre recombinase under the control of the $2 \mathrm{~kb} C r x$ promoter (Furukawa et al., 2002). We confirmed Cre-mediated recombination of a LacZ reporter transgene of the $C A G-C A T-Z$ Cre monitor mice in the developing and mature photoreceptor layer, and also confirmed the cell fate change of photoreceptor precursors by mating with the Ot $x 2^{f l o x}$ mice as previously reported (Nishida et al., 2003). All procedures conformed to the ARVO (Association for Research in Vision and Ophthalmology) Statement for the Use of Animals in Ophthalmic and Vision Research, and were approved by the Institutional Safety Committee on Recombinant DNA Experiments and the Animal Research Committee of Osaka Bioscience Institute. Mice were housed in a temperature-controlled room with a $12 \mathrm{~h}$ light/ dark cycle. Fresh water and rodent diet were available at all times.

Immunohistochemistry. Mouse eyes of either sex were fixed in $4 \%$ paraformaldehyde in PBS for $30 \mathrm{~s}$ or $5 \mathrm{~min}$, embedded in TissueTek OCT compound 4583 (Sakura Finetek), frozen, and sectioned. Frozen $20 \mu \mathrm{m}$ sections on slides were dried for $30 \mathrm{~min}$ at room temperature, rehydrated in PBS for $5 \mathrm{~min}$, incubated with blocking solution (5\% normal goat serum and $0.5 \%$ Triton $\mathrm{X}-100$ in $\mathrm{PBS}$ ) for $1 \mathrm{~h}$, and then with primary antibodies for $4 \mathrm{~h}$ at room temperature. Slides were washed with PBS three times for $10 \mathrm{~min}$ each time and incubated with secondary antibodies for $2 \mathrm{~h}$ at room temperature. The specimens were observed under a 
laser confocal microscope (LSM510, Carl Zeiss). We used the following primary antibodies: mouse monoclonal antibodies specific to CtBP2 (C-terminal binding protein 2) (1: 1000, BD Biosciences), dystrophin (1:300, Millipore Bioscience Research Reagents MAB1692), and PKC (1:1000, Sigma P5704); rabbit polyclonal antibodies to $\beta$-dystroglycan H-242 (1:300, Santa Cruz Biotechnology), TRPM1(1:500) (Koike et al., 2010) and Pikachurin (1:500, Wako Chemicals 011-22631) (Sato et al., 2008); and a guinea pig polyclonal antibody against mGluR6 (1:3000) (Koike et al., 2010). We used Cy3-conjugated secondary antibodies (1:400, Jackson ImmunoResearch Laboratories) and Alexa Fluor 488-conjugated secondary antibodies (1:400, Sigma).

$E R G$ recordings. Electroretinographic recordings were performed as described previously (Chen et al., 2004). In brief, 2- to 6-month-old mice of either sex were darkadapted overnight, and then anesthetized with an intramuscular injection of $70 \mathrm{mg} / \mathrm{kg}$ ketamine and $14 \mathrm{mg} / \mathrm{kg}$ xylazine. ERGs were recorded with a gold-wire loop electrode placed on the anesthetized cornea. The mice were placed in a Ganzfeld bowl and stimulated with stroboscopic stimuli of $1.0 \mathrm{log} \mathrm{cd}-\mathrm{s} / \mathrm{m}^{2}$ (photopic units) maximum intensity. Four levels of stimulus intensities ranging from -5.0 to 1.0 $\log \mathrm{cd}-\mathrm{s} / \mathrm{m}^{2}$ were used for the scotopic ERG recordings, and four levels of stimuli ranging from -0.5 to $1.0 \mathrm{log} \mathrm{cd}-\mathrm{s} / \mathrm{m}^{2}$ were used for the photopic ERGs. The photopic ERGs were recorded on a rod-suppressing white background of $1.3 \log \mathrm{cd}-\mathrm{s} / \mathrm{m}^{2}$.

Electron tomography. For observation by transmission electron microscope, 500-nmthick sections of the 2-month-old mouse retinas of either sex were cut with a diamond knife (Nanotome) using a Reichert Ultracut E ultramicrotome (Leica), and mounted on Formvarcoated 50 mesh square grids. Sections were stained as described previously (Takaoka et al., 2008). Colloidal gold beads ( $20 \mathrm{~nm}$; BB International), used as fiducial markers for alignment, were applied to the section surface. Sections were stabilized with a layer of evaporated carbon. Tomographic data were recorded automatically using an H-9500SD (Hitachi Co.) transmission electron microscope operated at an acceleration voltage of $300 \mathrm{kV}$. Data were recorded at a microscopic magnification of $15,000 \times$ (at $1.23 \mathrm{~nm} /$ pixel) from $-60^{\circ}$ to $+60^{\circ}$ at $2^{\circ}$ intervals around a single axis and collected with a $2048 \times$ 2048 CCD camera F224HD (Tietz Video and Imaging Processing Systems). For each tomography experiment, 5 and 15 different rod terminals from two wild-type and three mutant retinas, respectively, were observed. Image processing, including alignment and $3 \mathrm{D}$ reconstruction, was performed with the IMOD package as described previously (Kremer et al., 1996).

Cell culture and transfection. HEK293 cells were grown in DMEM (Sigma) with $10 \%$ fetal calf serum and $2 \mathrm{mg} / \mathrm{L}$ L-glutamine. Transfection was performed using Lipofectamine-LTX (Invitrogen) according to the manufacturer's instructions. At $72 \mathrm{~h}$ after transfection, cells were washed with PBS, fixed with 4\% paraformaldehyde in PBS for 3 min at room temperature, and subsequently incubated with blocking solution for $30 \mathrm{~min}$. Cells were immunostained with a primary antibody in the blocking solution for $4 \mathrm{~h}$ at room temperature, and subsequently incubated with the secondary antibody solution for $2 \mathrm{~h}$ at room temperature.
DG CKO
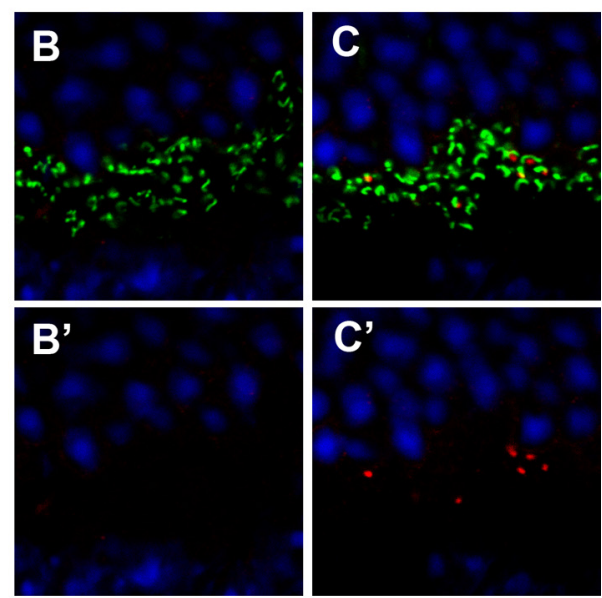

DG CKO

\section{Rod Cone}
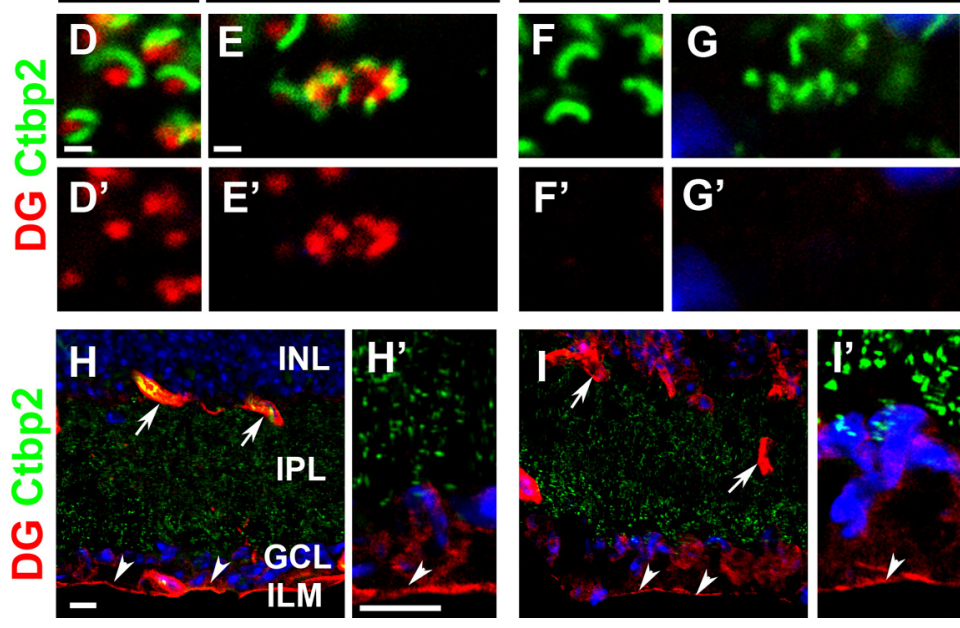

Figure 2. $D G$ in photoreceptor ribbon synapses is depleted in the $D G C K O$ retinas. Immunohistochemical analysis of $D G$ in the control and $D G C K O$ retinas. Retinal sections from control $\left(\boldsymbol{A}, \boldsymbol{A}^{\prime}, \mathbf{D}, \boldsymbol{D}^{\prime}, \boldsymbol{E}, \boldsymbol{E}^{\prime}, \boldsymbol{H}, \boldsymbol{H}^{\prime}\right)$ and $D G C K O\left(\boldsymbol{B}, \boldsymbol{B}^{\prime}, \boldsymbol{C}, \boldsymbol{C}^{\prime}, \boldsymbol{F}, \boldsymbol{F}^{\prime}, \mathbf{G}, \boldsymbol{G}^{\prime}, \boldsymbol{I}, \boldsymbol{I}^{\prime}\right)$ mice were stained with antibodies against DG (red) and CtBP2 (green), a synaptic ribbon marker in the ribbon synapse of the OPL. Nuclei sycer observed at the OPL in photoreceptors $(\boldsymbol{A})$, blood vessels ( $\operatorname{arrow}$ in $\boldsymbol{H}$ ), and the ILM ( $\boldsymbol{H}$, arrowheads). In the DG CKO retina, DG signal in the OPL is severely depleted $\left(\boldsymbol{B}, \boldsymbol{C}\right.$, whereas it remains in blood vessels (arrow in $I$ ) and the ILM (arrowheads in $I, I^{\prime}$ ). Scale bars: $\boldsymbol{A}, \boldsymbol{H}, \boldsymbol{H}^{\prime}, 10 \mu \mathrm{m} ; \boldsymbol{D}, \boldsymbol{E}, 1 \mu \mathrm{m}$. IPL, inner plexiform layer.

Western blot analysis. Transfected HEK293 cells were washed by PBS twice and lysed in a SDS-sample buffer. Western blot analysis was performed using a semidry transfer cell (iBlot system, Invitrogen) with iBlot Gel Transfer Stack PVDF (Invitrogen). Signals were detected using Can Get Signal (Toyobo) and ECL Plus Western Blotting Detection System (GE Healthcare Life Sciences).

\section{Results}

Dystrophin, DG, and Pikachurin are colocalized at retinal photoreceptor synaptic terminals

To investigate the subcellular localization of the DGC components at the synapses of developing retinal photoreceptors, we immunostained Pikachurin, DG, and dystrophin in the OPL where the photoreceptor, bipolar, and horizontal cell processes form ribbon synapses. Retinal sections from P4, P8, and P12 WT mice were stained with antibodies against Pikachurin, DG, dystrophin, and a synaptic ribbon marker, CtBP2 (Fig. 1A-I). At P4, 


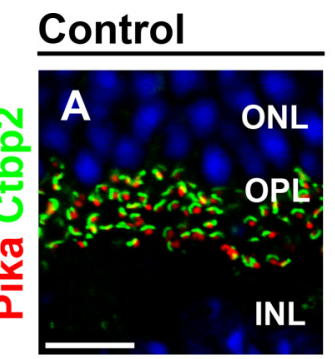

DG CKO
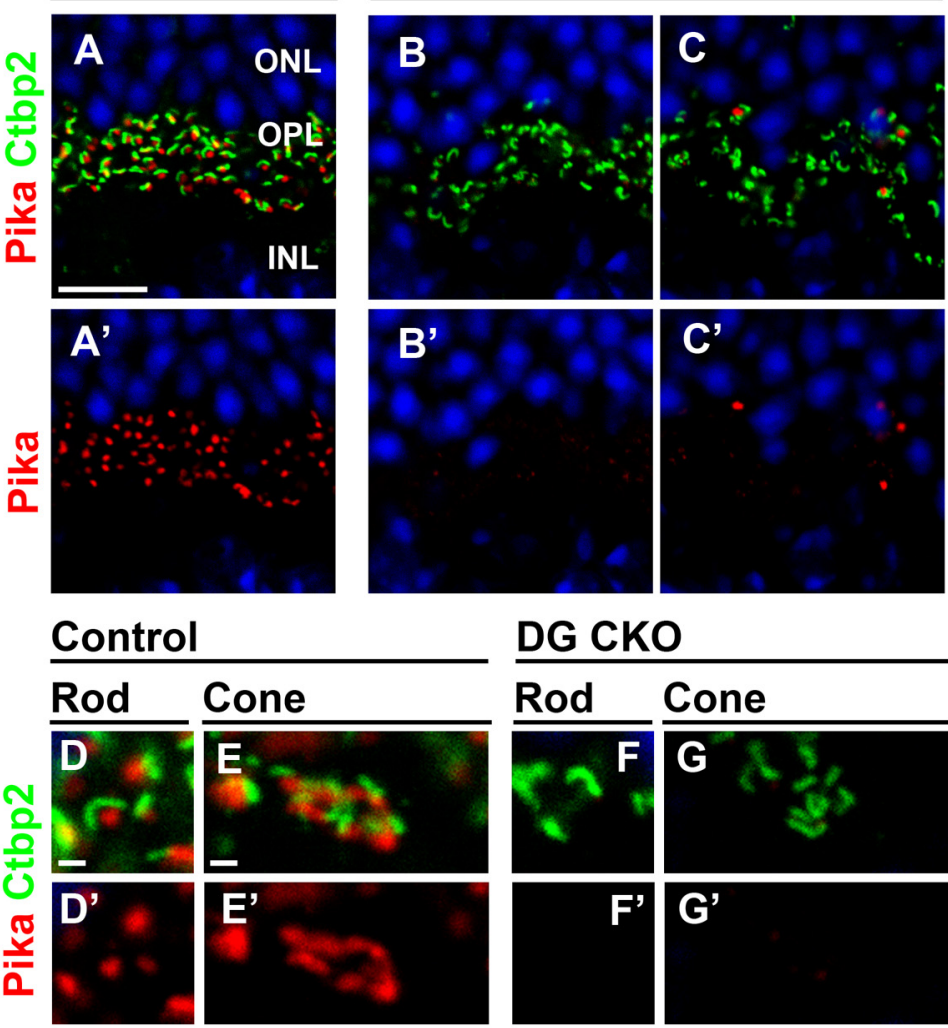

DG CKO
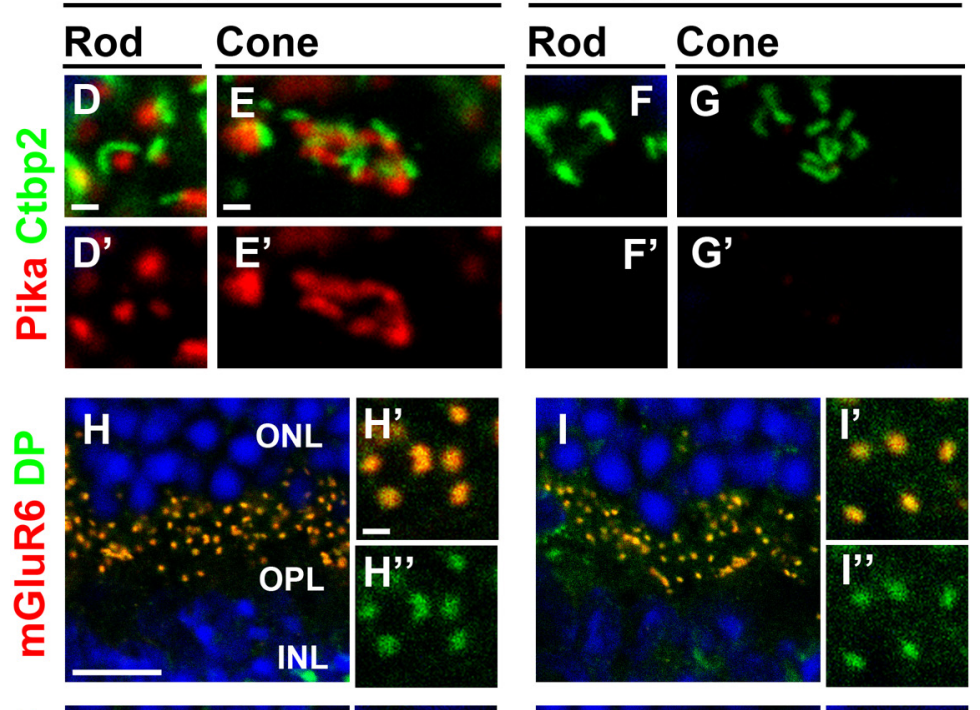

$\frac{0}{1}$
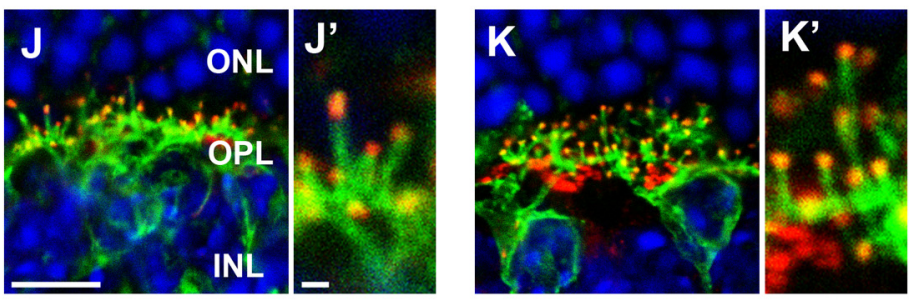

0
0
0
0
0
0
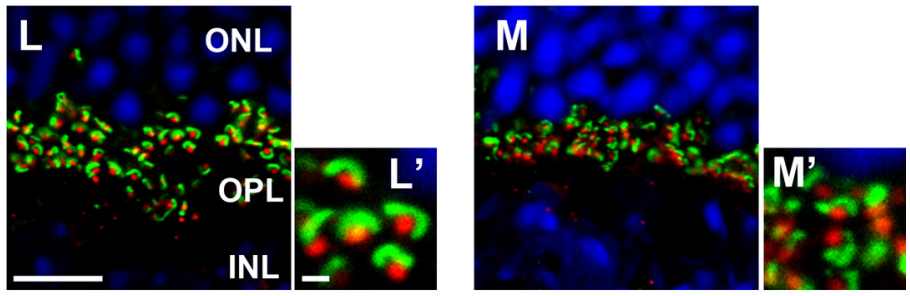

Figure 3. $D G$ is essential for the proper localization of Pikachurin in the photoreceptor ribbon synapse. Immunostaining of photoreceptor synaptic markers in the $D G C K 0$ retina. Retinal sections from control $\left(\boldsymbol{A}, \boldsymbol{A}^{\prime}, \boldsymbol{D}, \boldsymbol{D}^{\prime}, \boldsymbol{E}, \boldsymbol{E}^{\prime}, \boldsymbol{H}-\boldsymbol{H}^{\prime \prime}, \boldsymbol{J}, \boldsymbol{J}^{\prime}, \boldsymbol{L}, \boldsymbol{L}^{\prime}\right)$ and $D G C K 0$ mice $\left(\boldsymbol{B}, \boldsymbol{B}^{\prime}, \boldsymbol{C}, \boldsymbol{C}^{\prime}, \boldsymbol{F}, \boldsymbol{F}^{\prime}, \boldsymbol{G}, \boldsymbol{G}^{\prime}, \boldsymbol{I}-\boldsymbol{I}^{\prime \prime}, \boldsymbol{K}, \boldsymbol{K}^{\prime}, \boldsymbol{M}, \boldsymbol{M}^{\prime}\right)$ were stained with antibodies against Pikachurin (red in $\boldsymbol{A}-\boldsymbol{G}^{\prime}$ ), CtBP2 (green in $\boldsymbol{A}-\boldsymbol{G}^{\prime}, \boldsymbol{L}-\boldsymbol{M}^{\prime}$ ), dystrophin (green in $\boldsymbol{H}-\boldsymbol{I}^{\prime \prime}$ ), PKC (green in $\boldsymbol{J}-\boldsymbol{K}^{\prime}$; a marker for the dendrites of bipolar cells), mGluR6 (red in $\boldsymbol{H}, \boldsymbol{H}^{\prime}, \boldsymbol{I}$, $\left.\boldsymbol{I}^{\prime}, \boldsymbol{J}-\boldsymbol{M}^{\prime}\right)$. Nuclei were stained with DAPI (blue). Higher-magnification views of $\operatorname{rod}\left(\boldsymbol{D}, \boldsymbol{D}^{\prime}, \boldsymbol{F}, \boldsymbol{F}^{\prime}\right)$ and cone $\left(\boldsymbol{E}, \boldsymbol{E}^{\prime}, \boldsymbol{G}, \boldsymbol{G}^{\prime}\right)$ photoreceptor ribbon synapses were shown. Higher-magnification views of $\boldsymbol{H}, \boldsymbol{I}, \boldsymbol{J}, \boldsymbol{K}, \boldsymbol{L}$, and $\boldsymbol{M}$ are shown in $\boldsymbol{H}^{\prime}, \boldsymbol{H}^{\prime \prime}, \boldsymbol{I}^{\prime}, \boldsymbol{I}^{\prime \prime}, \boldsymbol{J}^{\prime}, \boldsymbol{K}^{\prime}, \boldsymbol{L}^{\prime}$, and $\boldsymbol{M}^{\prime}$, respectively. In the control retina, Pikachurin signals were found in both rod and cone photoreceptor synapses, however, in the $D G$ CKO retina, Pikachurin signals almost disappear. In contrast, the signals of dystrophin, mGluR6 and CtBP2 were undistinguishable between the control and DG CKO retinas. Scale bars: $\boldsymbol{A}, \boldsymbol{H}, \boldsymbol{J}, \boldsymbol{L}, 10 \mu \mathrm{m} ; \boldsymbol{D}, \boldsymbol{E}, \boldsymbol{H}^{\prime}, \boldsymbol{J}^{\prime}, \boldsymbol{L}^{\prime}, 1 \mu \mathrm{m}$. apposition of synaptic processes occurs, but synaptic processes have not invaginated into the photoreceptor axon terminals (Blanks et al., 1974). At this stage, we observed weak Pikachurin signals in the OPL adjacent to premature CtBP2positive ribbons (Fig. $1 D$ ). DG and dystrophin signals were less intense than the Pikachurin signal at this stage; however, these signals were weakly concentrated at the OPL (Fig. $1 A, D, G$ ). Around P7-P10, horizontal cell synaptic processes invaginate into the photoreceptor terminal, whereas bipolar processes have not invaginated (Blanks et al., 1974). At P8, obvious DG and Pikachurin signal puncta were observed adjacent to the synaptic ribbon (Fig. 1B,E). Strong dystrophin signals were also detected in the OPL (Fig. $1 H$ ). This result suggests that Pikachurin, DG, and dystrophin were concentrated in the photoreceptor synaptic terminals before the invagination of bipolar processes. Around P10-P14, the invagination of bipolar processes occurs and photoreceptor synaptic maturation is almost complete (Blanks et al., 1974). At P12, photoreceptor synapses with DG and Pikachurin puncta in a horseshoe-like ribbon similar to those of the adult retina were found (Fig. 1C,F). At this stage, obvious dystrophin signal puncta were also observed in the OPL (Fig. 1I). These results suggest that photoreceptor DGC formation occurs between P8 and P12, just before the invagination of bipolar dendritic tips. To confirm the precise localization of DG and dystrophin in the OPL, we immunostained DG and dystrophin with a glutamate receptor, mGluR6, which localizes specifically in ON-bipolar dendritic tips. We observed that DG and dystrophin signals almost completely overlapped the mGluR6 signals at the photoreceptor synapses in the OPL (Fig. $1 J-K^{\prime \prime}$ ). In addition, the signals of another ON-bipolar dendritic tip marker, TRPM1, also overlapped the DG and dystrophin signals (Fig. $1 L-M^{\prime \prime}$ ). These results suggest that DG and dystrophin localize at the invagination of the bipolar dendritic terminus in the photoreceptor synaptic terminus.

Photoreceptor-specific DG defect affects the synaptic localization of Pikachurin

To investigate DG function in the photoreceptor ribbon synapse, we ablated DG from photoreceptor cells by conditional gene targeting. To accomplish this, we mated the $D G^{f l o x / f l o x}$ line (Moore et al., 2002) with the Crx-Cre transgenic mouse line in which Cre-mediated recombina- 
tion occurs in both rod and cone photoreceptor precursors (Nishida et al., 2003). We generated $D G^{\text {flox/flox }} ;$ Crx-Cre (DG CKO) mice by mating $D G^{\text {flox/flox }}$ female mice with $D G^{\text {flox/flox }}$;Crx-Cre male mice. $D G$ CKO mice were viable and fertile without apparent developmental abnormalities in the retina. To confirm the loss of DG in photoreceptors, retinal sections from adult control $\left(D G^{\text {flox/flox }}\right)$ and $D G$ CKO (DG $G^{\text {flox/flox }}$; Crx-Cre) were stained with antibodies against $\beta$-DG and $\mathrm{CtBP} 2$ (Fig. $2 A-C^{\prime}$ ). In the control retina, DGpositive puncta were observed in the OPL (Fig. $2 A, A^{\prime}$ ). These DG signals were detected adjacent to horseshoe-like synaptic ribbons stained with $\mathrm{CtBP} 2$ (Fig. $2 A, A^{\prime}$ ). In the $D G \mathrm{CKO}$ retina, although the CtBP2 signal was observed in the OPL, the DG signal was below detection level in most of the OPL (percentage of $\mathrm{DG}^{+}$ $\mathrm{CtBP}^{+}{ }^{+}$synapses in $\mathrm{CtBP} 2{ }^{+}$synapses, $97.1 \pm 1.9 \%$ in control, $n=170$ from three animals, $10.7 \pm 4.4 \%$ in $D G$ CKO, $n=137$ from three animals; $p<0.01$ ) (Fig. $2 B-C^{\prime}$ ). We observed a few DGpositive synapses remaining in the OPL (Fig. $2 C, C^{\prime}$ ). In the mouse retina, $\sim 97 \%$ of the photoreceptors were rod photoreceptors (Carter-Dawson and LaVail, 1979). A rod photoreceptor synaptic terminus contains a single ribbon (Fig. $2 D, F)$, whereas a cone photoreceptor synapse contains a cluster of multiple ribbons (Fig. 2E, $G$ ). In the control retina, DG puncta were observed adjacent to both rod single ribbons (Fig. $2 D, D^{\prime}$ ) and cone ribbon clusters (Fig. $2 E, E^{\prime}$ ). In the $D G$ CKO retina, no significant DG signal was observed adjacent to the rod single ribbon (Fig. $2 F, F^{\prime}$ ) or cone ribbon clusters (Fig. 2G, $G^{\prime}$ ).

In the normal retina, DG is also observed in the Müller glial endfeet abutting the ILM (Fig. $2 H, H^{\prime}$ arrowheads) and perivascular glial endfeet (Fig. $2 \mathrm{H}$, arrows) (Schmitz and Drenckhahn, 1997b; Ueda et al., 2000; Blank et al., 2002). In contrast to the severe loss of DG in the OPL, DG signals remain in blood vessels (Fig. $2 I$, arrows) and the ILM (Fig. $2 I, I^{\prime}$ arrowheads) in the DG CKO retina. These results show that the loss of DG occurs specifically in rod and cone photoreceptors in the DG CKO retina.

Previous biochemical analysis showed that DG physically interacts with Pikachurin (Sato et al., 2008; Kanagawa et al., 2010). To examine whether the DG loss affects Pikachurin localization in photoreceptor synapses, we stained Pikachurin in adult control and DG CKO retinas. In the control retina, Pikachurin specifically localizes to the OPL in a punctate pattern adjacent to the synaptic ribbon stained with the anti-CtBP2 antibody in both rod and cone photoreceptor synapses (Fig. $3 A, D, E$ ). In contrast, the $D G$ CKO retina showed an obvious loss of the Pikachurin signal in most of the OPL (percentage of Pikachurin ${ }^{+} \mathrm{CtBP} 2{ }^{+}$synapses in $\mathrm{CtBP} 2{ }^{+}$synapses, $97.7 \pm 1.9 \%$ in control, $n=204$ from
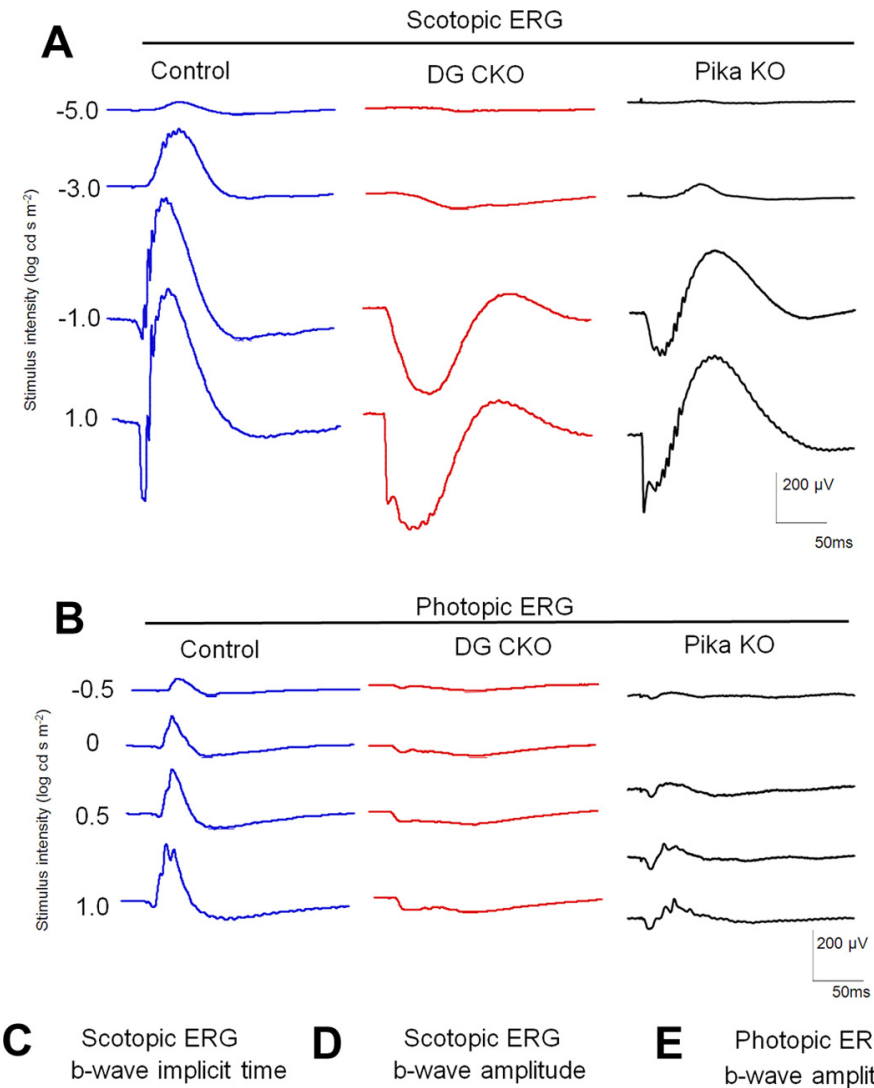

E Photopic ERG b-wave amplitude

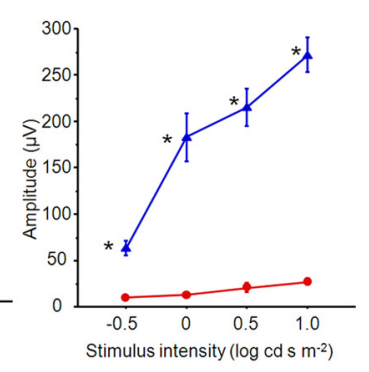

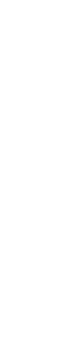

Figure 4. Electroretinograms recorded from DG CKO mice. Scotopic $(\boldsymbol{A})$ and photopic $(\boldsymbol{B})$ ERGs of an adult control mouse (left) trol $(n=3)$ and red lines indicate DGCKO $(n=6)$. DG CKO mice showed decreased amplitude under both scotopic and photopic conditions and prolongation of b-wave under scotopic conditions. The error bars indicate SEM. Asterisks indicate that the differences are statistically significant (Mann-Whitney test, $p<0.05$ ).

four animals, $7.5 \pm 4.8 \%$ in $D G$ CKO, $n=143$ from four animals; $p<0.01$ ) (Fig. $3 B-C^{\prime}, F-G^{\prime}$ ), although a few Pikachurin puncta remain in the OPL (Fig. $3 C, C^{\prime}$ ) as was observed with the DG signals (Fig. $2 C, C^{\prime}$ ). The absence of the Pikachurin signal is observed in both rod and cone photoreceptor synaptic terminals (Fig. $3 F-G^{\prime}$ ). This result suggests that DG in photoreceptors is essential for the synaptic localization of Pikachurin in photoreceptors. To investigate whether the deficiency of DG affects the integrity of bipolar dendritic structure, we performed immunostaining for mGluR6 and PKC, a marker for the ON-bipolar dendritic process. We found that mGluR6 signals accumulated at the tips of PKC-positive bipolar dendritic processes in both the control and $D G C K O$ retinas (Fig. $3 J-K^{\prime}$ ). In the normal retina, bipolar dendritic tips stained with mGluR6 localized adjacent to ribbons stained with CtBP2 (Fig. $3 L, L^{\prime}$ ). Similarly, we found that the mGluR6 signal was observed adjacent to synaptic ribbons in 
the $D G$ CKO retina (percentage of $\mathrm{mGluR6}^{+} \mathrm{CtBP}^{+}$synapses in $\mathrm{CtBP} 2{ }^{+}$synapses, $96.4 \pm 1.6 \%$ in control, $n=143$ from three animals, $92.1 \pm 9.7 \%$ in $D G$ CKO,$n=65$ from three animals; $p>$ 0.05 ) (Fig. $3 M, M^{\prime}$ ). These results suggest that a deficiency of DG does not affect the gross structure of bipolar dendritic tips. DG directly interacts with dystrophin and forms the DGC in photoreceptor synapses. We next examined whether the loss of DG affects the distribution of dystrophin in the OPL. We observed the dystrophin signal colocalizing with mGluR6 in the OPL of control mice (Fig. $\left.3 H-H^{\prime \prime}\right)$. Unexpectedly, we observed a similar distribution of dystrophin in the DG CKO retina (percentage of dystrophin ${ }^{+}$mGluR6 $^{+}$ puncta in mGluR6 ${ }^{+}$puncta, $100 \%$ in control, $n=191$ from four animals, $100 \%$ in DGCKO, $n=207$ from four animals) (Fig. $3 I-I^{\prime \prime}$ ). Since dystrophin also interacts with the other DGC components and actin cytoskeleton, these interactions may be enough to keep dystrophin in the photoreceptor synaptic terminal without DG.

\section{Loss of DG in photoreceptors affects synaptic signal} transmission between photoreceptor and bipolar cells

Loss of Pikachurin causes the attenuation of ERG activity, and a reduced and prolonged $\mathrm{b}$-wave in both scotopic and photopic conditions (Sato et al., 2008). Physical interaction between DG and Pikachurin in vitro suggests that DG plays an essential role in establishing a physiological connection between the photoreceptor terminus and bipolar dendrites (Sato et al., 2008; Kanagawa et al., 2010). To evaluate the physiological role of DG in the retina, we measured ERGs of adult $D G C K O$ mice. We observed that $D G$ $\mathrm{CKO}$ mice showed a decreased amplitude and prolonged $\mathrm{b}$-wave under scotopic conditions (Fig. $4 A, C, D$ ). We found a decreased b-wave amplitude under photopic conditions (Fig. $4 B, E$ ). The ERG b-wave responses are mainly generated by the activity of ON-bipolar cells (Robson and Frishman, 1995, 1996; Koike et al., 2010). These results suggest an essential function of DG in the physiological connection between photoreceptors and $\mathrm{ON}$ bipolar cells at ribbon synapses. Furthermore, we compared the degree of abnormality in ERGs between DG CKO and Pikachurin KO mice (Sato et al., 2008). Notably, the prolongation of b-wave implicit time under scotopic conditions was significantly more in the DG CKO mouse than in the Pikachurin KO mouse (Fig. 4A) (Sato et al., 2008). In addition, the b-wave amplitude under both photopic and scotopic conditions is less in the DG CKO mouse than in the Pikachurin $\mathrm{KO}$ mouse. These results indicate that loss of DG causes a more severe defect of synaptic connection between photoreceptor and bipolar cells than that caused by the loss of Pikachurin only.

\section{Electron microscopic analysis of photoreceptor ribbon synapses in the DG CKO mice}

To examine whether the loss of DG affects the ultrastructure of photoreceptor synapses, we performed 3D electron microscopic analyses on the adult control and DG CKO retinas. In this approach, projection images of longitudinal sections of photoreceptors were collected by tilting the specimen incrementally around an axis perpendicular to the electron beam. Using IMOD software, tomographic volumes $(2.5 \times 2.5 \times 0.5 \mu \mathrm{m})$ of the rod synaptic terminus that included a large portion of the synapse ribbon were calculated from the series of projection images. We analyzed tomographic slices of 3D volumes and 3D models in the control and DG CKO photoreceptor synapses (Fig. 5A-F). A normal rod synaptic terminus contains a single synaptic ribbon (Fig. 5A, labeled R), two horizontal cell processes (Fig. 5A, labeled $\mathrm{H}$ ), and two rod bipolar dendrites (Fig. 5A, labeled B). In the photoreceptor ribbon synapse of the control mouse, the ribbon is
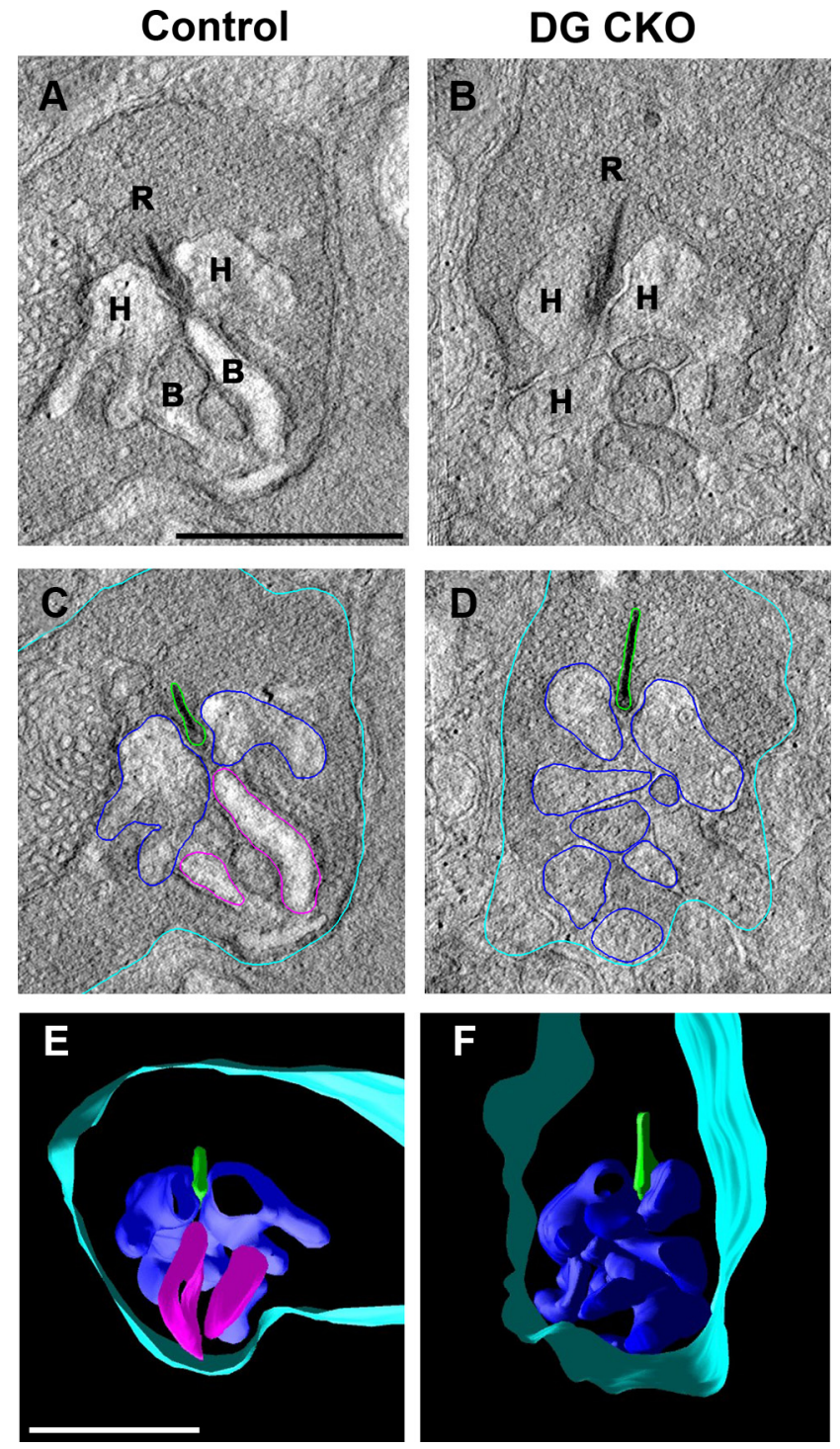

Figure 5. Electron microscopic analysis of the photoreceptor ribbon synapse in the DG CKO mice. Electron tomography of rod photoreceptor synapse terminals in the control $(A, C, E)$ and $D G C K O(B, D, F)$ retinas by ultrahigh-voltage electron microscopy. Synaptic ribbon (R), horizontal cell processes $(H)$, and bipolar cell dendrites $(B)$ are indicated in $\boldsymbol{A}$ and $\boldsymbol{B}$. Representative demarcation of bipolar dendritic tips (magenta), horizontal processes (dark blue), ribbon (green), and rod plasma membrane (light blue) for tomography are shown for control $(\boldsymbol{C})$ and $D G C K O(D)$ retinas. Bipolar cells dendrites do not appose to the synaptic terminal in the rod photoreceptor ribbon synapse in the $D G C K 0$ retina $(\boldsymbol{B}, \boldsymbol{D}, \boldsymbol{F})$. Scale bar represents $1 \mu \mathrm{m}(\boldsymbol{A}, \boldsymbol{E})$.

located at the apex of an invagination, which accommodates the postsynaptic processes of bipolar and horizontal cells (Fig. 5A). We observed that dendritic terminals of horizontal cells were normally inserted into the invagination of the photoreceptor ribbon synapse in both the control and DG CKO retinas (Fig, 5A$D)$. In contrast, we found that the dendritic processes of bipolar cells were not inserted into the invagination of the rod photoreceptor ribbon synapses in the $D G$ CKO retina (Fig. $5 B, D, F$ ). We also confirmed this using $3 \mathrm{D}$ models extracted from the tomograms (Fig. $5 E, F$ ). These results suggest that the loss of DG causes a structural defect of the synaptic connection between photoreceptors and bipolar cells. This structural defect of ribbon synapses in the $D G$ CKO retina may underlie the abnormality of the ERG b-wave observed in the DG CKO mice. 
Pikachurin is important for the proper assembly of DG in the photoreceptor ribbon synapse

We found that DG is essential for the proper localization of Pikachurin in the photoreceptor synapse. Conversely, is Pikachurin necessary for the normal localization of DG? To address this issue, we examined the localization of DG in the adult Pikachurin ${ }^{-1-}$ retina (Fig. $6 A-N^{\prime}$ ). In the WT retina, Pikachurin localizes adjacent to synaptic ribbon (Fig. 6A-A"). We confirmed that the Pikachurin signal is completely lost in the Pikachurin $^{-1-}$ retina (percentage of Pikachurin ${ }^{+} \mathrm{CtBP} 2{ }^{+}$synapses in $\mathrm{CtBP} 2{ }^{+}$synapses, $96.7 \pm 1.3 \%$ in WT, $n=121$ from three animals, $0 \%$ in Pikachurin ${ }^{-1-}, n=$ 154 from three animals; $p<0.01$ ) (Fig. $6 B-$ $B^{\prime \prime}$ ) (Sato et al., 2008). Interestingly, we found that the DG signal in the OPL is severely depleted in Pikachurin ${ }^{-1-}$ photoreceptor synapses compared with that in the control retina (percentage of $\mathrm{DG}^{+}$ $\mathrm{CtBP}^{+}{ }^{+}$synapses in $\mathrm{CtBP} 2{ }^{+}$synapses, $95.7 \pm 2.8 \%$ in WT, $n=208$ from four animals, $15.5 \pm 6.6 \%$ in Pikachurin ${ }^{-/-}$, $n=153$ from five animals; $p<0.01$ ) (Fig. $6 C, D)$. The DG signal loss is observed in both rod and cone photoreceptor synapses of the Pikachurin ${ }^{-1-}$ retina compared with those of the control retina (Fig. $\left.6 E-H^{\prime}\right)$. We previously showed that Pikachurin is expressed exclusively in photoreceptor cells and not in Müller glial cells (Sato et al., 2008). Consistent with this, the DG signals in blood vessels and the ILM of Müller glial endfeet are indistinguishable between the wild-type and Pikachurin ${ }^{-1-}$ retinas (Fig. $6 I-J^{\prime}$ ), suggesting that the ERG abnormality observed in the Pikachurin $^{-1-}$ mouse was not due to the defect of the DGC in Müller glial cells. Similar to the DG CKO retina, other photoreceptor synaptic markers including dystrophin and mGluR6 were unaffected in the Pikachurin $^{-1-}$ retina (percentage of dystrophin $^{+}$mGluR6 $^{+}$puncta in mGluR6 ${ }^{+}$ puncta, $100 \%$ in WT, $n=211$ from four animals, $100 \%$ in Pikachurin $^{-/-}, n=205$ from five animals; percentage of mGluR6 ${ }^{+}$ $\mathrm{CtBP} 2{ }^{+}$synapses in $\mathrm{CtBP} 2{ }^{+}$synapses, $95.8 \pm$ $2.7 \%$ in WT, $n=156$ from three animals, $92.5 \pm 2.2 \%$ in Pikachurin $^{-/-}, n=111$ from three animals; $p>0.05$ ) (Fig. $6 K-N^{\prime}$ ). These results suggest that Pikachurin is required for the assembly of DG in photoreceptor synaptic terminals.

Pikachurin Laminin-G repeat domains are sufficient for DG clustering on the cell surface

In Pikachurin ${ }^{-/-}$photoreceptor ribbon synapses, we observed significantly de-
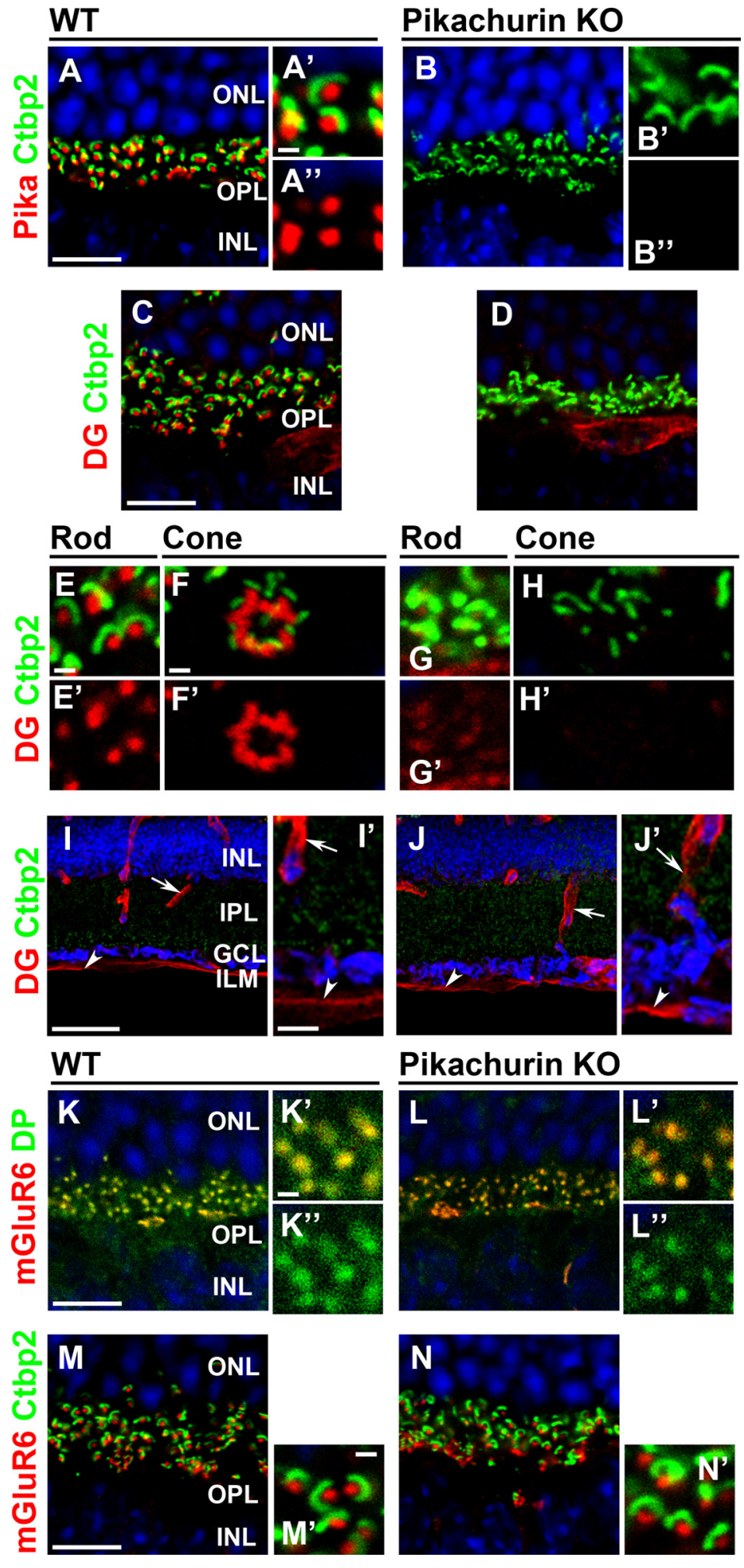

Figure 6. Pikachurin is required for the proper synaptic localization of DG in photoreceptor cells. Immunostaining of photoreceptor synaptic markers in the Pikachurin ${ }^{-/-}$retina. Retinal sections from control $\left(A-A^{\prime \prime}, C, E-F^{\prime}, I, I^{\prime}, K-K^{\prime \prime}, M, M^{\prime}\right)$ and Pikachurin $^{-1-}\left(\boldsymbol{B}-\boldsymbol{B}^{\prime \prime}, \mathbf{D}, \mathbf{G}-\boldsymbol{H}^{\prime}, \boldsymbol{J}, \boldsymbol{J}^{\prime}, \boldsymbol{L}-\boldsymbol{L}^{\prime \prime}, \boldsymbol{N}, \boldsymbol{N}^{\prime}\right)$ mice were stained with the antibodies against Pikachurin (red in $\left.\boldsymbol{A}-\boldsymbol{B}^{\prime \prime}\right)$, CtBP2 (green in $\boldsymbol{A}-\boldsymbol{J}^{\prime}, \boldsymbol{M}-\boldsymbol{N}^{\prime}$ ), DG (red in $\boldsymbol{C}-\boldsymbol{J}^{\prime}$ ), dystrophin (green in $\boldsymbol{K}-\boldsymbol{L}^{\prime \prime}$ ), and mGluR6 (red in $\boldsymbol{K}-\boldsymbol{N}^{\prime}$ ). Higher-magnification views of $\operatorname{rod}\left(\boldsymbol{E}, \boldsymbol{E}^{\prime}, \boldsymbol{G}, \boldsymbol{G}^{\prime}\right)$ and cone $\left(\boldsymbol{F}, \boldsymbol{F}^{\prime}, \boldsymbol{H}, \boldsymbol{H}^{\prime}\right)$ photoreceptor ribbon synapses were shown. Higher-magnification views of $\boldsymbol{A}, \boldsymbol{B}, \boldsymbol{I}, \boldsymbol{J}, \boldsymbol{K}, \boldsymbol{L}$, $M$, and $N$ are shown in $A^{\prime}, A^{\prime \prime}, B^{\prime}, B^{\prime \prime}, I^{\prime}, J^{\prime}, K^{\prime}, K^{\prime \prime}, L^{\prime}, L^{\prime \prime}, M^{\prime}$, and $\boldsymbol{N}^{\prime}$, respectively. Nuclei were stained with DAPI (blue). Loss of the Pikachurin signal in the Pikachurin ${ }^{-\prime-}$ OPL is shown $\left(\boldsymbol{B}-\boldsymbol{B}^{\prime \prime}\right)$. DG signal is significantly decreased in both rod and cone photoreceptor synapses of Pikachurin ${ }^{-/-}$mice $\left(\mathbf{G}-\boldsymbol{H}^{\prime}\right)$, whereas the DG in blood vessels (arrows in $\boldsymbol{I}_{\mathbf{I}} \mathbf{I}^{\prime}, \mathbf{J}, \mathbf{J}^{\prime}$ ) and the ILM (arrowheads in $I^{\prime} I^{\prime}, J, J^{\prime}$ ) are unaltered between WT and Pikachurin ${ }^{-\prime-}$ retinas. Dystrophin, mGluR6 and CtBP2 signals are indistinguishable between WT and Pikachurin ${ }^{-\prime-}$ retinas. Scale bars: $\boldsymbol{A}, \boldsymbol{C}, \boldsymbol{I}^{\prime}, \boldsymbol{K}, \boldsymbol{M}, 10 \mu \mathrm{m} ; \boldsymbol{I}, 50 \mu \mathrm{m} ; \boldsymbol{A}^{\prime}, \boldsymbol{E}, \boldsymbol{F}, \boldsymbol{K}^{\prime}, \boldsymbol{M}^{\prime}, 1 \mu \mathrm{m}$. IPL, inner plexiform layer. 


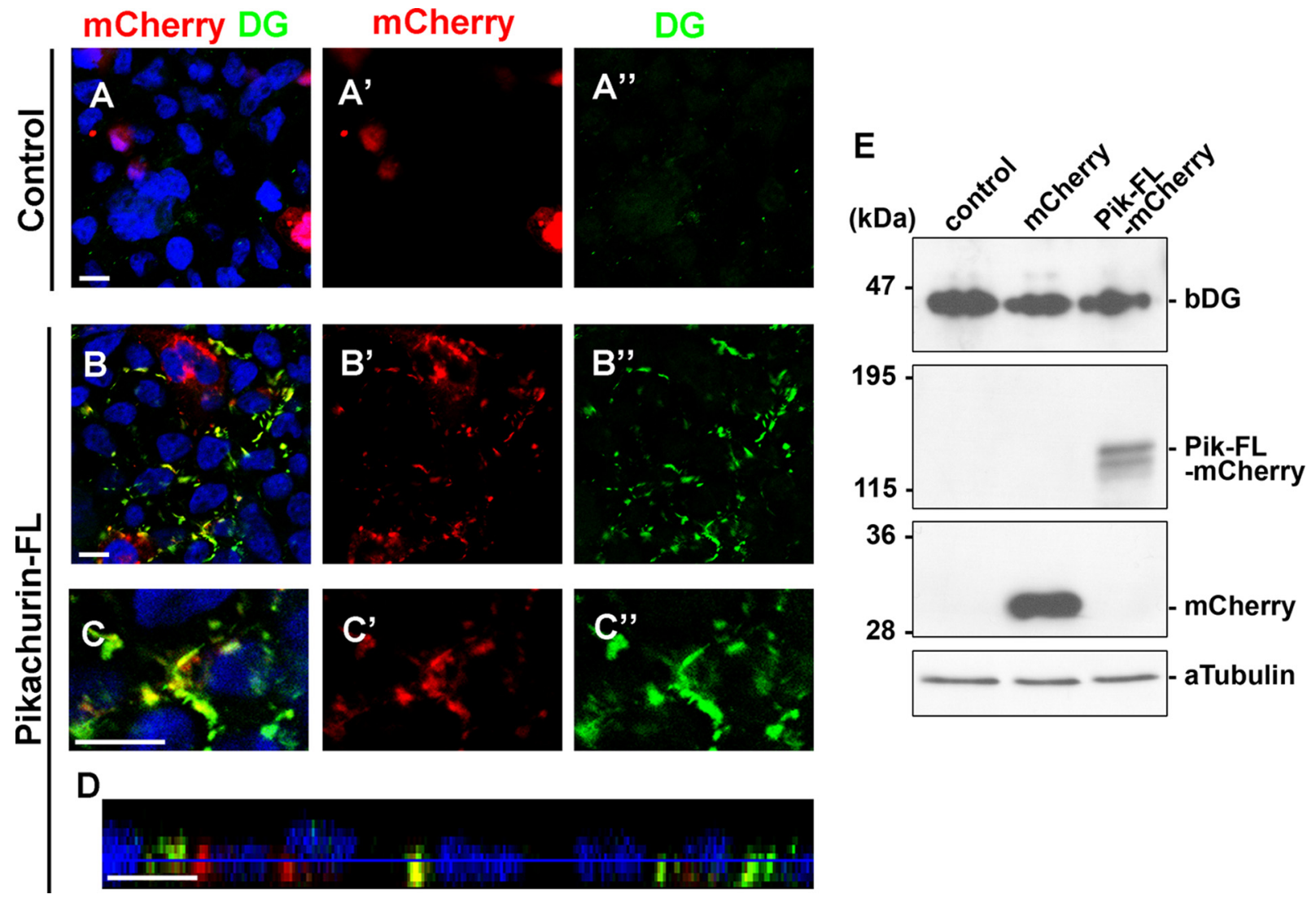

Figure 7. Overexpression of Pikachurin induces clustering of DG in HEK293 cells. HEK293 cells were transfected with a plasmid expressing mCherry (A-A') or Pikachurin-FL-mCherry (B-D). $\mathrm{mCherry}(\mathrm{red})$ and $\beta$-dystroglycan (DG, green) signals were observed. Transverse $\left(\boldsymbol{A}-\boldsymbol{C}^{\prime \prime}\right)$ or apical-basal $(\boldsymbol{D})$ optical sections of cells are shown. Higher-magnification views of HEK293 cells transfected with the Pikachurin-FL-mCherry plasmid are shown $\left(\boldsymbol{C}-\boldsymbol{C}^{\prime \prime}\right)$. DG clustering was observed at the cell-cell junction of cells transfected with the Pikachurin-FL-mCherry expression plasmid. Nuclei were stained with DAPI (blue). $\boldsymbol{E}$, Western blots were performed to examine the amount of DG in cells. No obvious change of DG amount was observed in the cells expressing Pikachurin or $\mathrm{mCherry}$ alone. Scale bars, $10 \mu \mathrm{m}$.

creased DG signals. This result suggests that Pikachurin is necessary for the proper assembly of DG in the photoreceptor ribbon synapse. Pikachurin may regulate the DG clustering on cell surfaces through direct interaction. To examine this possibility, we overexpressed Pikachurin in HEK293 cells, which endogenously express DG, and observed the localization change of endogenous DG by immunostaining (Fig. 7A-D). HEK293 cells were transfected with plasmids expressing $m$ Cherry tagged with full-length Pikachurin (Pikachurin-FL-mCherry) or mCherry only as a control, and stained with an anti-DG antibody. In the cells transfected with a control plasmid, we observed no obvious accumulation of the DG signal (Fig. 7A-A"). However, in the cells transfected with plasmids expressing Pikachurin-FL-mCherry, we found a concentrated DG signal in cell-cell junctions (Fig. 7B$D)$. Notably, an mCherry-tagged Pikachurin signal was enriched and clustered in cell-cell junctions together with the DG signal (Fig. $7 B-D$ ). This result shows that Pikachurin is sufficient to assemble DG on the cell surface. At the same time, this result suggests that the expression of Pikachurin induces the formation of the DG-Pikachurin complex on the cell surface. To test whether the total DG amount in the cells changes or not, we performed Western blots and analyzed the amount of DG in the cells. We observed no obvious change of DG protein amount between the cells transfected with the mCherry control and Pikachurin constructs (Fig. 7E). Overexpression of Pikachurin does not seem to induce the expression or degradation of DG.
To determine the critical domain of the Pikachurin protein for DG clustering on the cell surface, we prepared plasmids expressing various Pikachurin deletion constructs and transfected them into HEK293 cells (Fig. 8 A-I). We observed significant DG clusterings in the cells transfected with plasmids expressing Pikachurin deletion constructs with LG2-3 domains (Pik-FL, Pik-LG1-3, Pik-LG2-3, Pik-FN-LG) (Fig. $8 B-E^{\prime}$ ), whereas no DG clustering was detected in the cells transfected with plasmids expressing Pikachurin without the LG2-3 domains (Pik-FN, Pik-LG3, SSmCherry) (Fig. $8 F-H^{\prime}$ ). This result suggests that the Pikachurin LG2-3 domains are necessary to induce DG clustering on the cell surface, and that the LG2-3 domains were core regions for the formation of the DG-Pikachurin complex.

\section{Discussion}

Photoreceptor DG is required for normal retinal physiology In the current study, we investigated the functional role of DG in the retinal photoreceptor synaptic terminus. Attenuation of ERGs is observed in both MD patients and the corresponding model animals. In the mouse retina, DG is expressed in photoreceptor ribbon synapses in the OPL and Müller glial endfeet in the ILM and blood vessels (Schmitz and Drenckhahn, 1997b; Ueda et al., 2000; Blank et al., 2002). Since DG is detected in multiple areas of the retina, it was unclear which part of the defect is responsible for the ERG abnormality in MD patients and MD model animals. A recent study reported that mice with condi- 
tional deletions of DG in Müller glial cells using Nestin-Cre and GFAP-Cre showed a loss of DG from the ILM but maintained DG expression in the OPL (Satz et al., 2009). These mice showed weakly reduced ERG b-wave amplitudes, but no obvious prolongation of b-wave implicit time was observed (Satz et al., 2009). Although previous studies reported that DG and/or Pikachurin-positive puncta were reduced in DG CKO/Nestin-Cre mice, it is still unclear whether or not the photoreceptorspecific loss of DG causes physiological dysfunction (Satz et al., 2009; Hu et al., 2011). In this study, we demonstrated that the mice lacking DG in photoreceptors showed a severe reduction of ERG b-wave amplitude with marked prolongation of b-wave implicit time, suggesting that photoreceptor synaptic DG is critical for both the amplitude and implicit time of the b-wave. In addition, we also showed that deficiency of Pikachurin does not cause the loss of DG from Müller glial endfeet. This result also supports evidence that the loss of the DGC in the OPL can cause attenuation of ERGs, since Pikachurin ${ }^{-/-}$mice also showed ERG attenuation (Sato et al., 2008). Our results in this study and observations from previous studies suggest that prolonged b-wave implicit time in MD model mice is most likely due to the functional defect of DGC formation in photoreceptor synapses. However, our study did not exclude the possibility that the DGC defect expressed in cells other than photoreceptors contributes to the ERG attenuation observed in MD patients and MD animal models.

Several studies by immunoelectron microscopic analysis have shown that DG localizes in photoreceptor presynapses but not in the postsynaptic dendritic tips of bipolar or horizontal cells (Schmitz and Drenckhahn, 1997a; Ueda et al., 1998; Blank et al., 1999; Jastrow et al., 2006). We observed a loss of invagination in bipolar processes but not in horizontal processes in $D G$ CKO photoreceptor synapses. Why does the DG defect not affect the structure of horizontal processes? One possibility is that DG has a redundant factor in the horizontal process invagination, but not in the formation of bipolar processes. In mice, invagination of horizontal processes into photoreceptor synapses occurs between P7 and P10, whereas invagination of bipolar processes begins later, mainly between P10 and P14 (Blanks et al., 1974). A redundant factor for DG may function around P7-P10 in horizontal process invagination. Another possibility is that the molecular mechanism of invagination is totally different for horizontal and bipolar processes. An unknown molecular system independent from the DG-Pikachurin system may regulate horizontal process invagination.

I

Pik-FL

Pik-FN

Pik-LG3

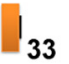

SS-mCherry

33
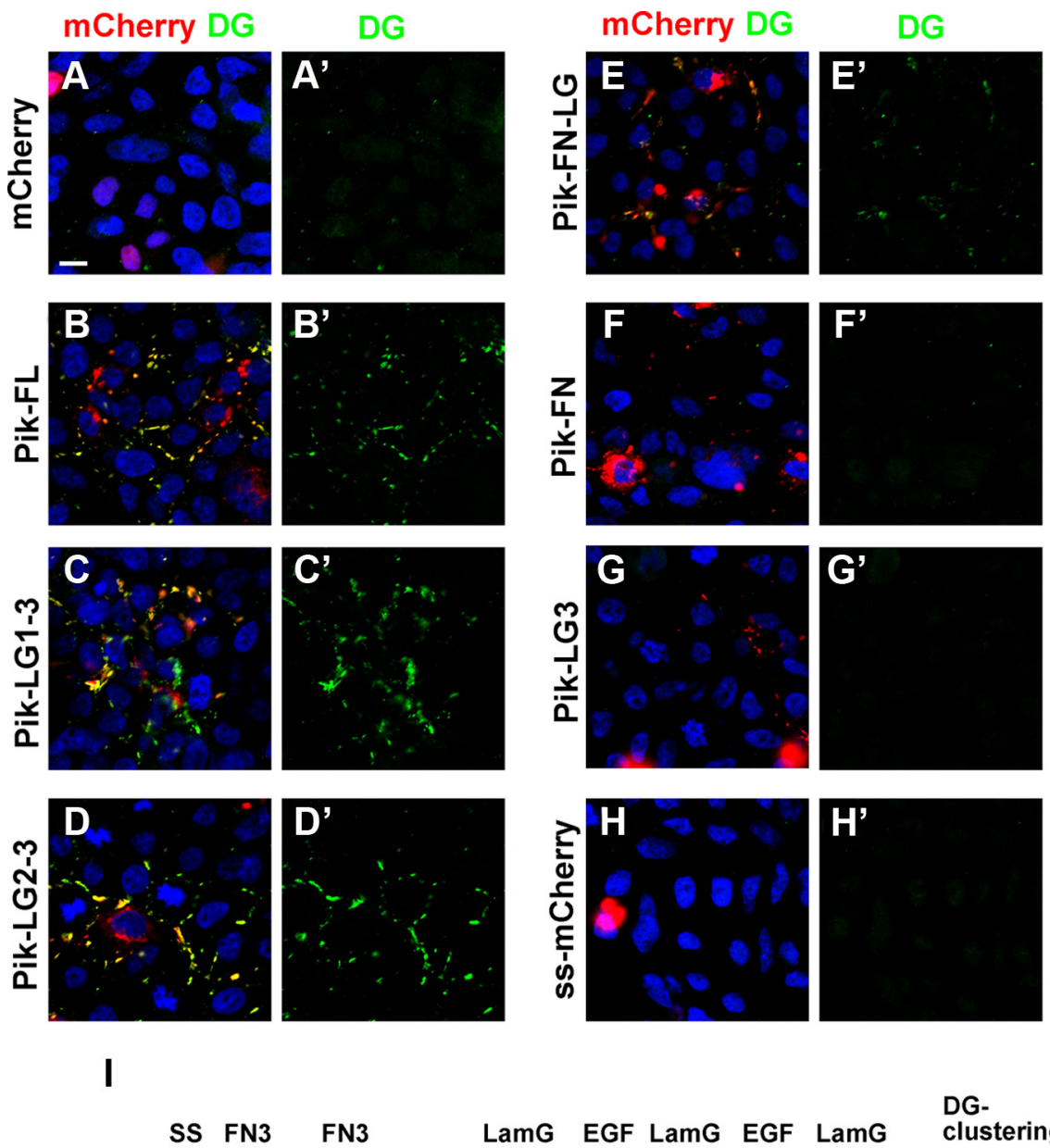

LamG EGF LamG EGF LamG

DG-
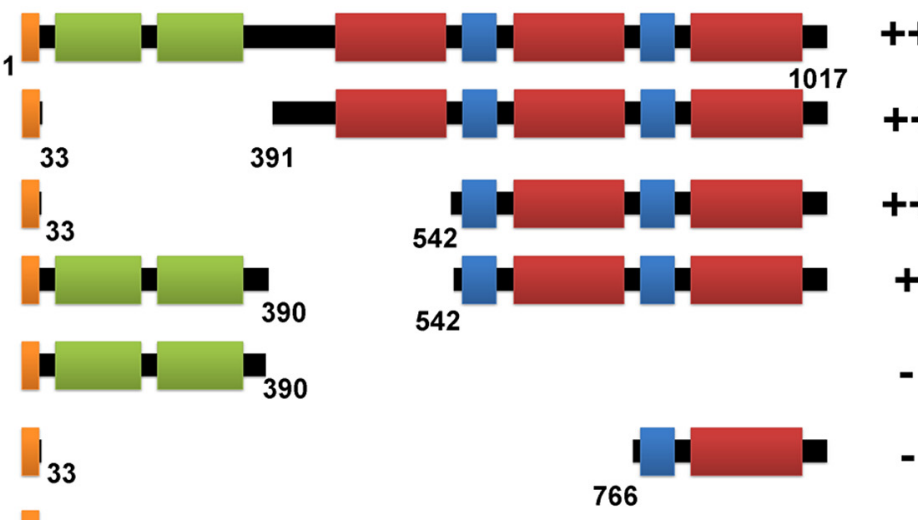

Figure 8. Laminin G repeats of Pikachurin are essential for the assembly of the DG-Pikachurin complex on the cell surface. $\boldsymbol{A}-\boldsymbol{H}^{\prime}$, HEK293 cells were transfected with plasmids expressing various Pikachurin-deletion constructs. Seventy-two hours after transfection, subcellular localization of DG (green) and mCherry-tagged Pikachurin-deletion proteins (red) was observed. Nuclei were stained with DAPI (blue). Scale bar represents $10 \mu \mathrm{m}$. I, A schematic diagram of Pikachurin deletion constructs fused with $\mathrm{mCherry}$ at their C terminus. Amino acid numbers are shown at the bottom of each construct. DG clustering was observed in cells transfected with plasmids expressing Pikachurin deletion constructs with LG2-3 domains (Pik-FL, Pik-LG1-3, Pik-LG2-3, Pik-FN-LG), whereas no DG clustering was detected in the cells transfected with plasmids expressing Pikachurin constructs without LG2-3 domains.

\section{Interaction of Pikachurin with DG induces DG clustering on} the cell surface

Previous biochemical studies showed a direct interaction of DG and Pikachurin in vitro (Sato et al., 2008; Kanagawa et al., 2010), however, their functional interaction in vivo had not been well examined. In the current study, we provided several lines of evidence for the interaction between Pikachurin and DG in vivo. 


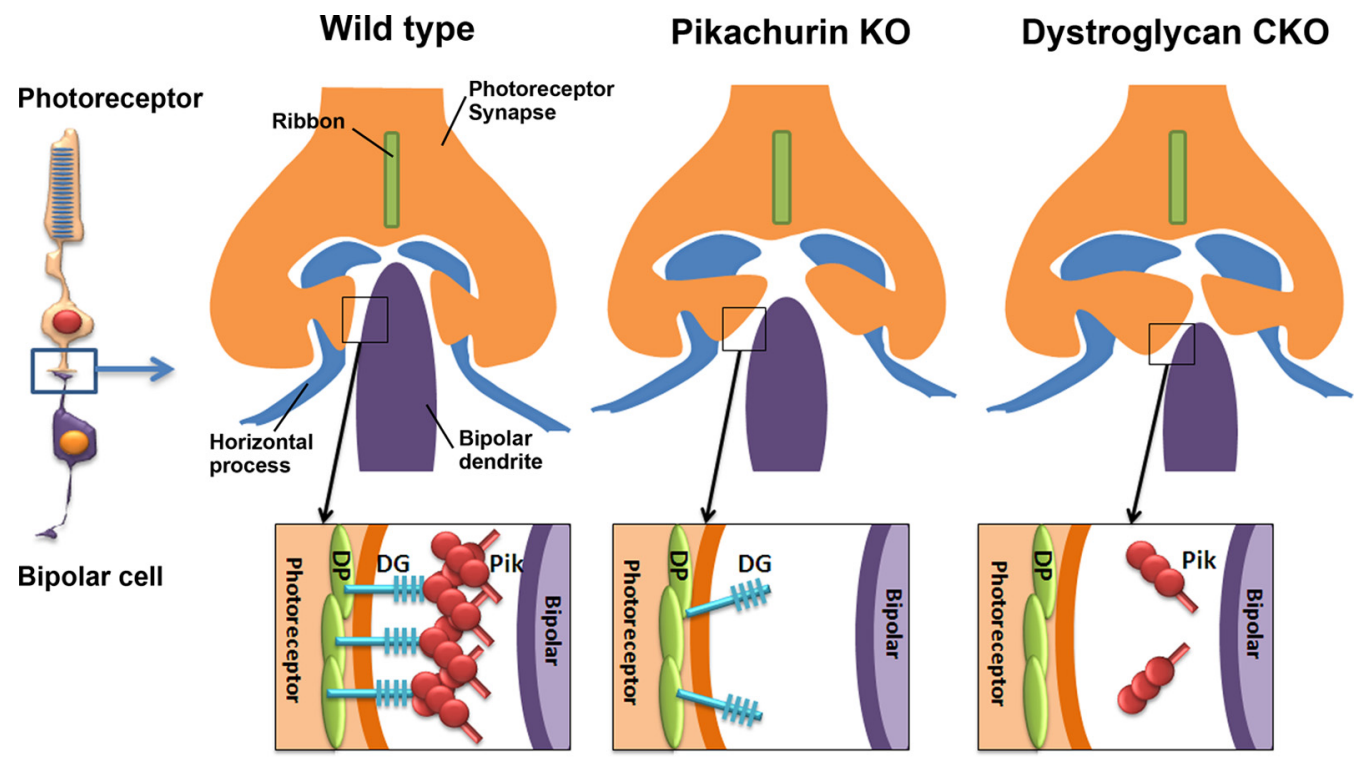

Figure 9. A hypothetical model of DG and Pikachurin function in photoreceptor synaptic terminals. Schematic diagrams of synaptic structures (top) and molecular interaction (bottom) in the WT (left), Pikachurin KO (middle) and DG CKO (right) retinas are shown. Left, In the WT retina, Pikachurin molecules form oligomers through their Laminin G domains. Oligomerization of Pikachurin induces the clustering of DG on the photoreceptor synaptic surface of the WT retina. Middle, In the Pikachurin ${ }^{-1-}$ retina DG clustering at the synaptic terminal is not induced because of the loss of DG interaction with oligomerized Pikachurin. Lack of DG clustering at the photoreceptor synaptic tip causes a defect of synaptic connection between photoreceptors and bipolar cells, resulting in the abnormal ERGs observed in the Pikachurin ${ }^{-\prime-}$ mice. Right, In the DG CKO retina, loss of DG prevents Pikachurin accumulation on the tips of photoreceptor synapses. Lack of DG in the synaptic terminal causes a more severe defect in the structure of photoreceptor synapses.

First, conditional deletion of DG in the photoreceptor presynaptic region causes a defect of Pikachurin localization in photoreceptor synapses, the prolongation of both ERG amplitude and implicit time, and a defect of synaptic ultrastructures between photoreceptors and bipolar cells. Second, the loss of Pikachurin affects proper DG assembly in the photoreceptor ribbon synapse. Third, expression of the DG binding domains of Pikachurin in cultured cells causes the clustering of DG on the cell surface. These results strongly support the idea that the interaction between DG and Pikachurin is essential for the formation of proper synaptic structure of photoreceptors.

In this study, we found that the forced expression of Pikachurin in cultured cells causes DG clustering on the cell surface. This result suggests that the interaction between Pikachurin and DG triggers assembly of the DG-Pikachurin complex on the cell surface. What is the molecular mechanism for the formation of this complex? One possibility is that Pikachurin oligomerization induces the assembly of the DG-Pikachurin complex. Previously we showed that Pikachurin is able to form oligomeric structures through LG repeats (Kanagawa et al., 2010). This suggests a molecular mechanism underlying the clustering of the DG-Pikachurin complex in the photoreceptor synapse; both the oligomerization of Pikachurin and the interaction of Pikachurin with DG cause the clustering of the DG-Pikachurin complex on the cell surface, thus, the DG-Pikachurin complex accumulates on the cell surface (Fig. 9). Interestingly, other DG interacting proteins including agrin, laminin, perlecan and neurexin also contain LG/ EGF repeats (Ervasti and Campbell, 1993; Gee et al., 1994; Peng et al., 1998; Sugita et al., 2001). DG clustering by DG-interacting proteins may be regulated by a common molecular mechanism through the LG repeat domains.

Notably, the abnormality of both amplitudes and implicit times of ERGs in the DGCKO mice were more severe than in the Pikachurin ${ }^{-1-}$ mice, suggesting that DG is more critical for photoreceptor synapse formation than Pikachurin. Consistent with our hypothesis, the DG signal was significantly reduced in pho- toreceptor synapses in the Pikachurin ${ }^{-/-}$OPL. However, a slight amount of DG remains in photoreceptor synapses in the Pikachu$\mathrm{rin}^{-1-}$ retina. There are two possible explanations for the phenotype difference between the loss of DG and the loss of Pikachurin. The first possible explanation is that DG is relatively stable even without interacting with Pikachurin. In this case, the structural change of the photoreceptor synapse caused by the loss of Pikachurin may be milder than that caused by the loss of DG, resulting in a milder ERG attenuation in the Pikachurin ${ }^{-1-}$ mice than that in the DG CKO mice. The second explanation is that DG also interacts with other extracellular matrix proteins in addition to Pikachurin, and this additional interaction partially keeps DG anchored on the photoreceptor synaptic surface without the DG-Pikachurin interaction. DG in the photoreceptor synapse may interact with other DG ligands including laminin, agrin, neurexin, perlecan, or uncharacterized ECM proteins.

In Drosophila, a Laminin G repeats-containing protein, spacemaker, is required for the generation of inter-rhabdomeral space, a small extracellular space among photoreceptor rhabdomers in the compound eyes (Zelhof et al., 2006). Interestingly, the amino acid sequence of the spacemaker Laminin $\mathrm{G}$ repeats is very similar to that of Pikachurin. It is possible that Pikachurin-like Laminin $\mathrm{G}$ domains may have the ability to expand and/or keep small extracellular space, such as photoreceptor synaptic cleft and inter-rhabdomeral space by oligomerization or interaction with other ECM proteins. Our observations in the current study suggest that the DG-Pikachurin complex regulates the formation of the fine structures of the synaptic terminal, what we called "micromorphogenesis" of the synaptic terminal.

A recent report showed that postsynaptic DG regulates presynaptic neurotransmitter release at the Drosophila NMJ (Bogdanik et al., 2008). Together with our observation, the loss of DG might affect the structure not only on the presynaptic side where DG localizes, but also on the postsynaptic side. Although we observed no obvious defects of mGluR6 distribution in the postsynapse of bipolar dendritic terminals in the OPL, we do not 
exclude the possibility that the loss of DG or Pikachurin affects the structure and/or the molecular composition of the postsynapse of bipolar terminals.

In summary, our findings demonstrate that photoreceptor DG is essential for the structural formation of the photoreceptor synapse and physiological synaptic connection between photoreceptor and bipolar cells. In addition, the loss of DG notably decreased the Pikachurin localization in photoreceptor synapses. We found that loss of Pikachurin in the photoreceptor synapse causes the depletion of DG at photoreceptor synapses. Furthermore, we observed that overexpression of Pikachurin in cultured cells induces formation of the DG-Pikachurin complex and facilitates the clustering of DG on the cell surface. These results suggest that Pikachurin is necessary and sufficient for DG assembly on the cell membrane, and for the functional link between Pikachurin and DG in photoreceptor synaptic formation. The micromorphogenesis mechanism observed in the DG-Pikachurin interaction in the photoreceptor-bipolar synapse formation may also be observed in the interaction between other LGcontaining DG ligands and DG in other synapses and tissues.

\section{References}

Beltrán-Valero de Bernabé D, Currier S, Steinbrecher A, Celli J, van Beusekom E, van der Zwaag B, Kayserili H, Merlini L, Chitayat D, Dobyns WB, Cormand B, Lehesjoki AE, Cruces J, Voit T, Walsh CA, van Bokhoven $\mathrm{H}$, Brunner HG (2002) Mutations in the O-mannosyltransferase gene POMT1 give rise to the severe neuronal migration disorder WalkerWarburg syndrome. Am J Hum Genet 71:1033-1043.

Blank M, Koulen P, Blake DJ, Kröger S (1999) Dystrophin and betadystroglycan in photoreceptor terminals from normal and $\mathrm{mdx} 3 \mathrm{Cv}$ mouse retinae. Eur J Neurosci 11:2121-2133.

Blank M, Blake DJ, Kröger S (2002) Molecular diversity of the dystrophinlike protein complex in the developing and adult avian retina. Neuroscience 111:259-273.

Blanks JC, Adinolfi AM, Lolley RN (1974) Synaptogenesis in the photoreceptor terminal of the mouse retina. J Comp Neurol 156:81-93.

Bogdanik L, Framery B, Frölich A, Franco B, Mornet D, Bockaert J, Sigrist SJ, Grau Y, Parmentier ML (2008) Muscle dystroglycan organizes the postsynapse and regulates presynaptic neurotransmitter release at the Drosophila neuromuscular junction. PLoS One 3:e2084.

Brockington M, Blake DJ, Prandini P, Brown SC, Torelli S, Benson MA, Ponting CP, Estournet B, Romero NB, Mercuri E, Voit T, Sewry CA, Guicheney P, Muntoni F (2001) Mutations in the fukutin-related protein gene (FKRP) cause a form of congenital muscular dystrophy with secondary laminin alpha2 deficiency and abnormal glycosylation of alpha-dystroglycan. Am J Hum Genet 69:1198-1209.

Carter-Dawson LD, LaVail MM (1979) Rods and cones in the mouse retina. I. Structural analysis using light and electron microscopy. J Comp Neurol 188:245-262.

Chen S, Kadomatsu K, Kondo M, Toyama Y, Toshimori K, Ueno S, Miyake Y, Muramatsu T (2004) Effects of flanking genes on the phenotypes of mice deficient in basigin/CD147. Biochem Biophys Res Commun 324:147-153.

Cibis GW, Fitzgerald KM, Harris DJ, Rothberg PG, Rupani M (1993) The effects of dystrophin gene mutations on the ERG in mice and humans. Invest Ophthalmol Vis Sci 34:3646-3652.

D’Souza VN, Nguyen TM, Morris GE, Karges W, Pillers DA, Ray PN (1995) A novel dystrophin isoform is required for normal retinal electrophysiology. Hum Mol Genet 4:837-842.

Ervasti JM, Campbell KP (1993) A role for the dystrophin-glycoprotein complex as a transmembrane linker between laminin and actin. J Cell Biol 122:809-823.

Fitzgerald KM, Cibis GW, Giambrone SA, Harris DJ (1994) Retinal signal transmission in Duchenne muscular dystrophy: evidence for dysfunction in the photoreceptor/depolarizing bipolar cell pathway. J Clin Invest 93:2425-2430.

Furukawa A, Koike C, Lippincott P, Cepko CL, Furukawa T (2002) The mouse Crx $5^{\prime}$-upstream transgene sequence directs cell-specific and developmentally regulated expression in retinal photoreceptor cells. J Neurosci 22:1640-1647.
Gee SH, Montanaro F, Lindenbaum MH, Carbonetto S (1994) Dystroglycanalpha, a dystrophin-associated glycoprotein, is a functional agrin receptor. Cell 77:675-686.

Haenggi T, Fritschy JM (2006) Role of dystrophin and utrophin for assembly and function of the dystrophin glycoprotein complex in non-muscle tissue. Cell Mol Life Sci 63:1614-1631.

Henry MD, Campbell KP (1996) Dystroglycan: an extracellular matrix receptor linked to the cytoskeleton. Curr Opin Cell Biol 8:625-631.

Hoffman EP, Kunkel LM (1989) Dystrophin abnormalities in Duchenne/ Becker muscular dystrophy. Neuron 2:1019-1029.

Hu H, Li J, Zhang Z, Yu M (2011) Pikachurin interaction with dystroglycan is diminished by defective $O$-mannosyl glycosylation in congenital muscular dystrophy models and rescued by LARGE overexpression. Neurosci Lett 489:10-15.

Ibraghimov-Beskrovnaya O, Ervasti JM, Leveille CJ, Slaughter CA, Sernett SW, Campbell KP (1992) Primary structure of dystrophin-associated glycoproteins linking dystrophin to the extracellular matrix. Nature 355:696-702.

Jastrow H, Koulen P, Altrock WD, Kröger S (2006) Identification of a betadystroglycan immunoreactive subcompartment in photoreceptor terminals. Invest Ophthalmol Vis Sci 47:17-24.

Kanagawa M, Omori Y, Sato S, Kobayashi K, Miyagoe-Suzuki Y, Takeda S, Endo T, Furukawa T, Toda T (2010) Post-translational maturation of dystroglycan is necessary for pikachurin binding and ribbon synaptic localization. J Biol Chem 285:31208-31216.

Kobayashi K, Nakahori Y, Miyake M, Matsumura K, Kondo-Iida E, Nomura Y, Segawa M, Yoshioka M, Saito K, Osawa M, Hamano K, Sakakihara Y, Nonaka I, Nakagome Y, Kanazawa I, Nakamura Y, Tokunaga K, Toda T (1998) An ancient retrotransposal insertion causes Fukuyama-type congenital muscular dystrophy. Nature 394:388-392.

Koike C, Obara T, Uriu Y, Numata T, Sanuki R, Miyata K, Koyasu T, Ueno S, Funabiki K, Tani A, Ueda H, Kondo M, Mori Y, Tachibana M, Furukawa $\mathrm{T}$ (2010) TRPM1 is a component of the retinal ON bipolar cell transduction channel in the mGluR6 cascade. Proc Natl Acad Sci U S A 107:332-337

Kremer JR, Mastronarde DN, McIntosh JR (1996) Computer visualization of three-dimensional image data using IMOD. J Struct Biol 116:71-76.

Lee Y, Kameya S, Cox GA, Hsu J, Hicks W, Maddatu TP, Smith RS, Naggert JK, Peachey NS, Nishina PM (2005) Ocular abnormalities in Large(myd) and Large(vls) mice, spontaneous models for muscle, eye, and brain diseases. Mol Cell Neurosci 30:160-172.

Liu J, Ball SL, Yang Y, Mei P, Zhang L, Shi H, Kaminski HJ, Lemmon VP, Hu $\mathrm{H}$ (2006) A genetic model for muscle-eye-brain disease in mice lacking protein $\mathrm{O}$-mannose 1,2- $\mathrm{N}$-acetylglucosaminyltransferase (POMGnT1). Mech Dev 123:228-240.

Longman C, Brockington M, Torelli S, Jimenez-Mallebrera C, Kennedy C, Khalil N, Feng L, Saran RK, Voit T, Merlini L, Sewry CA, Brown SC, Muntoni F (2003) Mutations in the human LARGE gene cause MDC1D, a novel form of congenital muscular dystrophy with severe mental retardation and abnormal glycosylation of alpha-dystroglycan. Hum Mol Genet 12:2853-2861.

Moore SA, Saito F, Chen J, Michele DE, Henry MD, Messing A, Cohn RD, Ross-Barta SE, Westra S, Williamson RA, Hoshi T, Campbell KP (2002) Deletion of brain dystroglycan recapitulates aspects of congenital muscular dystrophy. Nature 418:422-425.

Nishida A, Furukawa A, Koike C, Tano Y, Aizawa S, Matsuo I, Furukawa T (2003) Otx2 homeobox gene controls retinal photoreceptor cell fate and pineal gland development. Nat Neurosci 6:1255-1263.

Peng HB, Ali AA, Daggett DF, Rauvala H, Hassell JR, Smalheiser NR (1998) The relationship between perlecan and dystroglycan and its implication in the formation of the neuromuscular junction. Cell Adhes Commun 5:475-489.

Pillers DA, Bulman DE, Weleber RG, Sigesmund DA, Musarella MA, Powell BR, Murphey WH, Westall C, Panton C, Becker LE, Worton RG, Ray PN (1993) Dystrophin expression in the human retina is required for normal function as defined by electroretinography. Nat Genet 4:82-86.

Pillers DA, Weleber RG, Woodward WR, Green DG, Chapman VM, Ray PN (1995) mdxCv3 mouse is a model for electroretinography of Duchenne/ Becker muscular dystrophy. Invest Ophthalmol Vis Sci 36:462-466.

Robson JG, Frishman LJ (1995) Response linearity and kinetics of the cat retina: the bipolar cell component of the dark-adapted electroretinogram. Vis Neurosci 12:837-850. 
Robson JG, Frishman LJ (1996) Photoreceptor and bipolar cell contributions to the cat electroretinogram: a kinetic model for the early part of the flash response. J Opt Soc Am A Opt Image Sci Vis 13:613-622.

Sato S, Omori Y, Katoh K, Kondo M, Kanagawa M, Miyata K, Funabiki K, Koyasu T, Kajimura N, Miyoshi T, Sawai H, Kobayashi K, Tani A, Toda T, Usukura J, Tano Y, Fujikado T, Furukawa T (2008) Pikachurin, a dystroglycan ligand, is essential for photoreceptor ribbon synapse formation. Nat Neurosci 11:923-931.

Satz JS, Philp AR, Nguyen H, Kusano H, Lee J, Turk R, Riker MJ, Hernández J, Weiss RM, Anderson MG, Mullins RF, Moore SA, Stone EM, Campbell KP (2009) Visual impairment in the absence of dystroglycan. J Neurosci 29:13136-13146.

Schmitz F, Drenckhahn D (1997a) Localization of dystrophin and betadystroglycan in bovine retinal photoreceptor processes extending into the postsynaptic dendritic complex. Histochem Cell Biol 108:249-255.

Schmitz F, Drenckhahn D (1997b) Dystrophin in the retina. Prog Neurobiol 53:547-560.

Sugita S, Saito F, Tang J, Satz J, Campbell K, Südhof TC (2001) A stoichiometric complex of neurexins and dystroglycan in brain. J Cell Biol 154:435-445.

Takaoka A, Hasegawa T, Yoshida K, Mori H (2008) Microscopic tomography with ultra-HVEM and applications. Ultramicroscopy 108:230-238. tom Dieck S, Brandstätter JH (2006) Ribbon synapses of the retina. Cell Tissue Res 326:339-346.

Ueda H, Gohdo T, Ohno S (1998) Beta-dystroglycan localization in the photoreceptor and Muller cells in the rat retina revealed by immunoelectron microscopy. J Histochem Cytochem 46:185-191.

Ueda H, Baba T, Ohno S (2000) Current knowledge of dystrophin and dystrophin-associated proteins in the retina. Histol Histopathol 15:753-760.

van Reeuwijk J, Janssen M, van den Elzen C, Beltran-Valero de Bernabé D, Sabatelli P, Merlini L, Boon M, Scheffer H, Brockington M, Muntoni F, Huynen MA, Verrips A, Walsh CA, Barth PG, Brunner HG, van Bokhoven H (2005) POMT2 mutations cause alpha-dystroglycan hypoglycosylation and Walker-Warburg syndrome. J Med Genet 42:907-912.

Yoshida A, Kobayashi K, Manya H, Taniguchi K, Kano H, Mizuno M, Inazu T, Mitsuhashi H, Takahashi S, Takeuchi M, Herrmann R, Straub V, Talim B, Voit T, Topaloglu H, Toda T, Endo T (2001) Muscular dystrophy and neuronal migration disorder caused by mutations in a glycosyltransferase, POMGnT1. Dev Cell 1:717-724.

Zelhof AC, Hardy RW, Becker A, Zuker CS (2006) Transforming the architecture of compound eyes. Nature 443:696-699. 